\title{
Diskurse als Ort der mathematischen Denkentwicklung - Eine interaktionistische Perspektive
}

\author{
Marcus Schütte $\mathbb{D} \cdot$ Judith Jung · Götz Krummheuer
}

Eingegangen: 21. Oktober 2019 / Angenommen: 2. März 2021 / Online publiziert: 7. April 2021

(C) Der/die Autor(en) 2021

Zusammenfassung Der folgende Beitrag befasst sich mit dem Mathematiklernen in der frühen Kindheit aus interaktionistischer Perspektive. Mathematiklernen wird hierzu in zwei Aspekte ausdifferenziert: den Erwerb mathematischer Begriffe und Prozeduren im Sinne des Lernens von mathematischen Inhalten und die mathematische Denkentwicklung im Sinne des Argumentierenlernens. Der Fokus dieses Beitrags liegt auf dem zweiten Aspekt. Basierend auf interaktionistischen Vorstellungen zum Lernen wird die mathematische Denkentwicklung als zunehmende Partizipation an mathematischen Diskursen beschrieben. Hierdurch rücken mathematische Diskurse als „Ort“ der mathematischen Denkentwicklung ins Zentrum der Betrachtung. Zur näheren Beschreibung dieser Diskurse wird die bis dato gängige Fokussierung interaktionistischer Ansätze des Mathematiklernens auf die Analyse mathematischer Bedeutungsaushandlungen um eine Beschreibung emergierender argumentativer und semantischer Strukturierungen mathematischer Aushandlungsprozesse erweitert. In den empirischen Analysen des Beitrages werden mithilfe dieser theoretischen Erweiterung unterschiedliche Diskursstile rekonstruiert, die wir als narrative, formale und narratorische Diskurse bezeichnen. In künftigen Arbeiten sollen sie als theoretische Grundlage für die longitudinale Rekonstruktion der Partizipation von Lernenden an mathematischen Aushandlungsprozessen zur Beschreibung ihrer mathematischen Denkentwicklung genutzt werden.

\footnotetext{
M. Schütte $(\bowtie)$

Leibniz Universität Hannover, Hannover, Deutschland

E-Mail: marcus.schuette@ifs.uni-hannover.de

J. Jung

Technische Universität Dresden, Dresden, Deutschland

G. Krummheuer

Kassel, Deutschland
} 
Schlüsselwörter Diskurs · Partizipation · Mathematische Denkentwicklung · Interaktionistische Perspektive

\section{Discourses as the Place for the Development of Mathematical Thinking}

Abstract The following article considers mathematics learning in the early childhood from an interactionist perspective. Here, mathematics learning is differentiated into two aspects of learning: the acquisition of mathematical terms and procedures in the sense of learning mathematical content, and the development of mathematical thinking in the sense of learning to reason. The focus of this contribution is on the second aspect. Based on interactionist perceptions of learning the development of mathematical thinking is described as an increasing participation in mathematical discourses. As a result, mathematical discourses as the "place" for the development of mathematical thinking move into the center of attention. For a more detailed description of these discourses, the so far common focus of interactionist approaches to mathematics learning on the analysis of mathematical negotiation of meaning is expanded to include a description of the emerging argumentative and semantic structuring of the mathematical negotiation processes. In the empirical analyses of this article, different discourse styles are reconstructed, which we call narrative, formal and 'narrational' discourses. In future work, they will be used as a theoretical basis for the longitudinal reconstruction of the participation of learners in mathematical negotiation processes to describe their development of mathematical thinking.

Keywords Discourse - Participation - Development of mathematical thinking · Interactionist perspective

\section{Einleitung: Mathematische Denkentwicklung aus interaktionistischer Perspektive}

Der folgende Beitrag fußt auf der Idee des zugrundeliegenden Forschungsvorhabens, die Entwicklung mathematischen Denkens von Kindern longitudinal zu beobachten und so eine Theorie mathematischer Denkentwicklung zu entwerfen. Mathematische Denkentwicklung wird von uns aus einer interaktionistischen Perspektive beschrieben und als eine von zwei Komponenten des Mathematiklernens verstanden. So differenzieren wir Mathematiklernen mit Bezug zu Bruners Ansatz zum frühen Mutterspracherwerb (Bruner 2002) analytisch in zwei Aspekte aus: den Erwerb mathematischer Begriffe und Prozeduren und die Entwicklung mathematischen Denkens im Sinne einer Einführung in eine mathematische Argumentationskultur (vgl. Schütte und Krummheuer 2017). Zur mathematischen Denkentwicklung liegen bereits zahlreiche psychologisch orientierte Arbeiten vor. Man denke bspw. an Arbeiten von Piaget (1972), welche einen Ausgangspunkt für zahlreiche weitere Forschungsbemühungen in diesem Bereich darstellen. Auch die Erforschung spezifischer mathematischer Inhaltsbereiche, wie der Erwerb der Zählkompetenz oder des räumlichen Denkens, scheint bereits grundlegend erfolgt zu sein wie z.B. durch Fuson (1988) oder Newcombe und Huttenlocher (2003). Das dem vorliegenden Bei- 
trag zu Grunde liegende Forschungsvorhaben setzt vor allem an drei Kritikpunkten bestehender Ansätze und einer aus ihnen resultierenden Erklärungslücke früher mathematischer Denkentwicklung aus interaktionistischer Perspektive an:

- Die Mathematik wird in Forschungen zur kindlichen Denkentwicklung meist ,nur als ein Anwendungsfall oder Anwendungsbeispiel für eine darzustellende universelle psychologische, eigentlich inhaltsneutrale Entwicklungstheorie verstanden.

- Es werden zumeist nur Prozesse zu einzelnen mathematischen Inhalten, wie z. B. dem Zählen oder der Orientierung im Raum, untersucht und das spezifische und umfassende Mathematische daran - die Fähigkeit auf der Basis von Erlerntem mathematisch zu handeln - wird wenig berücksichtigt.

- Die kindliche Denkentwicklung wird in diesen Forschungen theoretisch als ein innerpsychisch zu verortender Prozess modelliert. Gemeinsames Handeln und Reden mit Erwachsenen oder anderen Kindern wird in diesen Theorien dabei zumeist als ,kontingente Randbedingung“ der Denkentwicklung (Oevermann et al. 1976, S. 371), also als für das Verstehen vom ,Wesen“ der Denkentwicklung nicht notwendiges, sondern lediglich bedingendes Phänomen im Außen, begriffen (siehe auch Krummheuer 1992).

Ziel unserer Forschungsbemühungen ist es, die spezifischen ,Denkweisen “ in der Mathematik stärker zu betonen. Dabei soll das verbindende Element der frühen mathematischen Denkentwicklung über die unterschiedlichen mathematischen Inhaltsbereiche hinaus - die spezifisch mathematischen strukturellen Gemeinsamkeiten früher fachlicher Aushandlungsprozesse - herausgearbeitet werden.

In dem vorliegenden Beitrag werden erste Teilergebnisse aus unserem Forschungsvorhaben vorgestellt. Zur theoretischen Verortung sollen zunächst die Grundlagentheorien der eingenommen interaktionistischen Perspektive dargelegt werden (siehe Abschn. 2). Sie grenzt sich - als ergänzende Perspektive - von theoretischen Ansätzen ab, nach denen das Lernen von Mathematik bzw. die Entwicklung mathematischer Fähigkeiten als ein in erster Linie innerpsychisch zu verortender Prozess modelliert wird (vgl. dazu u. a. Ehm et al. 2017; Peter Koop und Grüßing 2007; Schneider et al. 2016). Die auf diesen Grundlagen aufbauende Interaktionstheorie des Mathematiklernens sieht die Interaktion mit anderen für das kindliche mathematische Lernen als ein unabdingbares Basiskonzept an. Mathematiklernen lässt sich hiernach als ein wechselseitig bezugnehmender Prozess von ,Außen“ - der Partizipation an Interaktionsprozessen - und ,Innen “ - den kognitiven Umstrukturierungen des Individuums - verstehen. Interaktionistische mathematikdidaktische Arbeiten fokussieren dabei vor allem auf die Analyse situativer Bedeutungsaushandlungen mathematischer Objekte bzw. Inhalte. Diese Fokussierung ermöglicht eine Beschreibung der grundlegenden sowie spezifischen Bedingungen der Möglichkeit des Mathematiklernens in Interaktionsprozessen (vgl. u. a. Fetzer 2007; Schütte 2009; Tiedemann 2012). Solche Ansätze stoßen jedoch an ihre Grenzen, längerfristige Entwicklungsprozesse einzelner Individuen zu beschreiben, weswegen die mathematische Denkentwicklung von einzelnen Kindern bis dato nicht explizit in ihrem Interesse stand (erste Ansätze hierzu finden sich bei Brandt 2004). 
Für unser Forschungsvorhaben bedarf es deshalb eines grundlegenden Perspektivwechsels auf das, was mathematische Denkentwicklung zu sein vermag sowie einer lokalen Theorieentwicklung (vgl. Kelle 1994), die die bestehende Interaktionstheorie des Mathematiklernens im Sinne einer Theorie mathematischer Denkentwicklung aus interaktionistischer Perspektive erweitert, um so Entwicklungsprozesse von einzelnen Kindern beschreibbar machen zu können (siehe Abschn. 3). Innerhalb dieser Erweiterung greifen wir das partizipationstheoretische Lernmodell des Mathematiklernens durch zunehmend autonomere Partizipation von Krummheuer und Brandt (2001) auf und modifizieren dieses für die Analyse mathematischer Denkentwicklung. Die so hergeleitete Annahme, dass sich die mathematische Denkentwicklung über die Form der Partizipation in mathematischen Diskursen rekonstruieren lässt, rückt den Diskurs als ,im Außen liegenden Ort ' der mathematischen Denkentwicklung in den Blick. Die hier vorgestellten ersten Teilergebnisse des Forschungsvorhabens beschäftigen sich mit der möglichen Spezifizierung dieses ,Ortes ‘ der mathematischen Denkentwicklung. Es werden mathematische Diskurse in Bezug auf ihre spezifischen argumentativen und semantischen Strukturierungen analysiert. Zwei der in diesem Zusammenhang rekonstruierten Diskursstile - der narrative und der formale Diskurs - werden empirisch hergeleitet und lassen sich in bereits bestehende Theoriekonzepte einordnen (siehe Abschn. 4.1). Der dritte Diskursstil - der narratorische Diskurs - wird in seinen Charakteristika aus der Empirie skizziert, bedarf aber zu seiner theoretischen Einordnung eines abduktiven Schlusses, der über das zuvor hinzugezogene Theoriegerüst hinausweist (siehe Abschn. 4.2). Wir sehen in der Ausarbeitung des diskursiven Charakters der mathematischen Denkentwicklung eine konsistente und vielversprechende Erweiterung bestehender theoretischer Ansätze. Mithilfe der Bestimmung der unterschiedlichen Diskursstile lässt sich der ,diskursive Anspruch' der jeweiligen Situation an Lernende für eine erfolgreiche Partizipation beschreiben und hiermit erste punktuelle Stationen auf dem Weg einer mathematischen Denkentwicklung festhalten. Es bleibt allerdings späteren Arbeiten des Autorenteams vorbehalten zu beschreiben, wie eine longitudinal zu beobachtende je spezifische Partizipation an mathematischen Diskursen zu einer individuellen mathematischen Denkentwicklung führt. Abgerundet wird der vorliegende Beitrag mit Überlegungen zu einer lerntheoretischen Einordnung der rekonstruierten Diskursstile sowie einem Ausblick über weitere zu bearbeitende Forschungsdesiderate (siehe Abschn. 5).

\section{Theoretische Grundlagen: Mathematiklernen in und durch Interaktion}

Unsere Entwicklung einer Theorie mathematischer Denkentwicklung von Kindern im Vorschul- und frühen Grundschulalter basiert auf interaktionistisch-konstruktivistischen Vorstellungen zur kognitiven Entwicklung des Individuums (vgl. Cobb und Bauersfeld 1995). Für eine theoretische Fundierung einer solchen Perspektive auf das Lernen und die mathematische Denkentwicklung bedarf es Grundlagentheorien, die das Soziale bzw. Kollektive und nicht das Individuelle bei der (frühen) Entwicklung des Menschen ins Zentrum stellen. Wesentliche theoretische Grundannahmen 
unserer Untersuchung finden sich in der soziologischen Theorie des Symbolischen Interaktionismus (Blumer 2013). Das aus dem Symbolischen Interaktionismus abgeleitete und hier zugrundliegende Lernverständnis versteht die soziale Interaktion als konstituierender Ausgangspunkt von Lernprozessen. Für Blumer wird das menschliche Zusammenleben durch symbolische Interaktionen konstituiert, welche sich dadurch charakterisieren lassen, dass in ihnen Handlungen stets auf der Basis von zuvor interpretierten Handlungen des Gegenübers ausgeführt werden. Der Begriff der Interpretation und die aus Interpretationen resultierende Bedeutung bilden die Kernelemente des Symbolischen Interaktionismus. Bedeutungen von Objekten können dieser Theorie folgend als ,,soziale Produkte, [...] Schöpfungen, die in den und durch die definierenden Aktivitäten miteinander interagierender Personen hervorgebracht werden“ bezeichnet werden (Blumer 2013, S. 67). Die individuellen Interpretationen, die von den Individuen im Rahmen des kollektiven Aushandlungsprozesses vorgenommen werden, lassen sich als ein stets in Veränderung befindender „internalisierter sozialer Prozess“ (Blumer 2013, S. 68) verstehen. Bedeutungen von Objekten und insbesondere solche, die eine situationsüberdauernde Geltung bekommen, sind demnach weder in den Objekten noch in den Subjekten per se enthalten, sondern werden in einem intersubjektiven Raum konstruiert. Das Individuum entwirft, verändert und passt die Bedeutung der Objekte in Abhängigkeit an die jeweilige soziale Situation an (vgl. Blumer 2013; Keller 2012). So wird der Würfel erst dadurch zum Würfel, dass die an der Interaktion beteiligten Personen seine Bedeutung als Würfel mit je spezifischen Eigenschaften wechselseitig aushandeln und ihn mit einer situationsüberdauernden Bezeichnung Würfel benennen.

Dies bedeutet auch, dass nicht nur das jeweilige mathematische Thema, sondern auch das Lernen selbst bzw. die Bedingungen für die Möglichkeiten des Mathematiklernens ${ }^{1}$ situationell gebunden sind (siehe auch Steinbring 2000). In fachlichen - hier mathematischen - Interaktionsprozessen erhält das Individuum die Möglichkeit an kollektiven Bedeutungskonstruktionen zu partizipieren, die seine individuellen Fähigkeiten der Bedeutungskonstruktion überschreiten und so die Grundlage für individuelles Lernen darstellen. Konkret lässt sich dieses Wechselspiel von Innen und Außen wie folgt beschreiben: Zu Beginn einer Interaktion entwerfen die Beteiligten auf Grundlage ihrer individuellen Erfahrungen und Kenntnisse erste vorläufige Deutungen von der Situation, in der sie sich befinden. Diese individuellen Situationsdefinitionen erfolgen aus interaktionistischer Perspektive in Antizipation möglicher Deutungen anderer Beteiligter und in Anpassung an die anderen in der Interaktion emergierenden Deutungen im Prozess gemeinsamer Bedeutungsaushandlungen. Mit dem Begriff Situationsdefinition ist somit nicht ein Produkt bzw. eine Definition, sondern ein nach vorne offener Prozess einer permanenten Deutungsaktivität gemeint. Der wechselseitige Abgleich der individuellen Situationsdefinitionen

\footnotetext{
1 Wir sprechen hier von Bedingungen der Möglichkeit des Lernens in Abgrenzung zu Bedingungen des Lernens. Diese Differenzierung soll dem Faktum Rechnung tragen, dass über das Ergreifen der geschaffenen Möglichkeiten zum Lernen das Individuum je nach individuellen Fähigkeiten und/oder Präferenzen entscheidet und eine Entscheidung zur Ablehnung also eine Entscheidung nicht zu lernen auch bei noch so optimierten Bedingungen möglich ist. Der Ausdruck ,Bedingungen des Lernens' suggeriert hingegen, dass man durch Schaffung dieser Bedingungen ein Lernen des Individuums unausweichlich hervorrufen könnte.
} 
zwischen den Beteiligten kann zur Hervorbringung einer als geteilt geltenden Deutung, einem Deutungsinterim ${ }^{2}$, führen (zum Begriff siehe auch Voigt 1994, S. 78; ,als geteilt geltende[s] ' Verständnis). Das Deutungsinterim ist sozial konstituiert und kann für das Individuum potenziell neuartig sein, wenn es die Deutungskapazität des Individuums systematisch überschreitet. Es stellt somit das ,Anregungspotential ‘ für situationsüberdauernde kognitive Konstruktions- und Umstrukturierungsprozesse des Individuums dar. In anderen Worten: Der sozialen Interaktion kommt eine Orientierungsfunktion für die individuellen kognitiven Konstruktions- und Umstrukturierungsprozesse zu.

Durch die in Interaktionen wiederholt ausgehandelten Deutungsinterims verändern und verfestigen sich die Situationsdefinitionen der Beteiligten. Die Situationsdefinitionen ,konvergieren' hiernach zu standardisierten und routinisiert aktivierbaren Situationsdefinitionen, die von einer Person in ähnlichen Situationen erneut hervorgebracht werden können. Diese situationsüberdauernden kognitiven Konstruktionen des Individuums werden als Rahmungen bezeichnet (Krummheuer 1992). Dies führt zu der Erkenntnis, dass mathematisches Lernen die situationsüberdauernde Neukonstruktion oder Modifikation von Rahmungen in Bezug auf mathematische Inhalte und Argumentationsweisen ist. Die Ebenen der Kognition und Interaktion scheinen an dieser Stelle zu verschwimmen, weil individuelle situationsüberdauernde kognitive Konstruktions- und Umstrukturierungsprozesse unausweichlich wechselseitig mit kollektiven mathematischen Aushandlungsprozessen verbunden sind (siehe bspw. auch das Konzept des Commognitive Framework von Sfard 2008). Aus interaktionistischer Perspektive lässt sich Mathematiklernen somit als ein Prozess verstehen, der ,dualistisch“ sowohl im Inneren eines Individuums als auch in den Interaktionsprozessen, an denen die Person Teil hat, verortet ist (siehe hierzu auch Voigt 1995). Die soziale Interaktion ist Ausgangspunkt des Mathematiklernens und geht den individuellen Prozessen voran.

In dem folgenden Unterkapitel wird das interaktionistische Verständnis von Lernen anhand zweier Modelle des Mathematiklernens - Lernen durch Partizipation an kollektiven Argumentationen und Lernen mit Formaten - welche durch Krummheuer (1992) in die mathematikdidaktische Diskussion eingeführt wurden, genauer beschrieben und ausdifferenziert. Mit ihnen lassen sich Bedingungen der Möglichkeiten des Lernens von Mathematik in Interaktionen beschreiben (vgl. Jung 2019; Jung und Schütte 2018).

\footnotetext{
${ }^{2}$ In älteren Arbeiten wird an dieser Stelle in Anlehnung an den Begriff ,,working consensus“ von Goffman (1959, S. 9) von Arbeitskonsensus bzw. Arbeitsinterim gesprochen (vgl. Krummheuer 1992, S. 25). Wir verwenden dafür nun den Begriff des Deutungsinterims, da er zwei als wesentlich angesehene Aspekte der als geteilt geltenden Deutung in einem Begriff vereint. Das erste Element des Kompositums verweist darauf, dass ein gemeinsamer Aushandlungsprozess vollzogen worden ist. Der zweite Teil des Kompositums soll darauf verweisen, dass es sich lediglich um etwas ,Zwischenzeitliches', etwas Interimistisches, handelt, welches als Basis für weitere Aushandlungsprozesse dient.
} 


\subsection{Zwei Modelle mathematischen Lernens: Kollektive Argumentation und Format}

Das erste Modell stellt, basierend auf Ausführungen von Miller (1986), den Begriff der Argumentation ins Zentrum der Betrachtung. So findet Miller (1986, S. 23) folgend individuelles Lernen von Neuem durch Partizipation an „kollektiven Argumentationen“, d.h. an interaktiven Aushandlungsprozessen von interindividuellen Koordinationsproblemen, statt. Diese Idee schließt an das sozial-konstruktivistische Konzept des „,sozio-kognitiven Konflikts“ (Miller 1986, S. 143; siehe auch Doise et al. 1975) an. In der Identifikation auftretender Differenzen zwischen verschiedenen Beteiligten eines Interaktionsprozesses und dem Versuch einer argumentativen Auflösung dieser Differenzen wird hiernach die grundlegende Möglichkeit für das Lernen von Neuem gesehen (vgl. Miller 1986; Nührenbörger und Schwarzkopf 2010; Schwarz et al. 2000). Krummheuer (1997) greift diesen Aspekt des Lernens durch Argumentation für die mathematikdidaktische Lernforschung auf. Im Gegensatz zum Verständnis von Miller (1986) muss für ihn die Hervorbringung einer kollektiven Argumentation jedoch nicht auf einem Konflikt unter den Beteiligten beruhen. So kann die Hervorbringung einer Argumentation aus einer ethnomethodologischen Perspektive auch ohne sich widersprechende Argumente durch die in den jeweiligen Handlungen angezeigten Sinnhaftigkeiten vollzogen werden (Krummheuer 1997). Unter einer kollektiven Argumentation wird im Weiteren das gemeinsame Hervorbringen einer Argumentation von mehreren Interaktionsteilnehmenden verstanden, in der vielfach argumentative Begründungen geliefert werden, auch ohne dass etwas explizit strittig sein muss. Dies ist z. B. der Fall, wenn die Lehrperson Lernende auffordert, ihre Antworten zu begründen und für alle zu erklären, selbst wenn von niemandem ein Widerspruch eingebracht wird und keine Streitigkeit vorliegt (vgl. Jung 2019).

Das zweite Modell zur Beschreibung von Bedingungen der Möglichkeiten des Mathematiklernens greift auf ein Konzept zum frühkindlichen Spracherwerb von Bruner (2002) zurück. Dieses Modell zeigt Parallelen zu dem sozial-konstruktivistischen Konzept der Zone der nächsten Entwicklung von Vygotsky (1969) auf und fußt auf der Idee des Lernens in einer Gruppe mit einem oder mehreren fachlich in der Interaktion fortgeschrittenen Beteiligten ${ }^{3}$. Bruner (2002, S. 15) folgend setzt ein erfolgreicher Verlauf des Mutterspracherwerbs die Existenz eines „Language Acquisition Support System“ (LASS) voraus. Mit LASS bezeichnet er die Gesamtheit der dialogischen Aktivitäten zwischen den in der Interaktion fortgeschrittenen Personen und dem Kind. Empirisch rekonstruiert Bruner als LASS wiederholt strukturgleich emergierende Interaktionsmuster - sogenannte „Formate“ (2002, S. 103) - in denen über die Zeit eine Rollenverschiebung zwischen Kind und Erwachsenem stattfindet, mit der das Kind zunehmend autonomere Handlungsanteile in der gemeinsamen

\footnotetext{
3 In der Interaktion fachlich fortgeschrittene Personen sind klassisch Interaktionspartnerinnen und -partner wie Lehrpersonen oder Eltern, die mit dem fachlichen Inhalt bereits vertraut sind und bewusst oder unbewusst in asynchronen Lehr-Lernsituationen mit Kindern stehen. Es können aber auch Mitlernende sein, die aufgrund bereits gemachter Erfahrungen mit dem spezifischen Lerngegenstand vertraut sind und so als fachlich in der Interaktion fortgeschritten zu betrachten sind.
} 
Interaktion übernimmt. In Analogie zu dem LASS entwirft Krummheuer (2011, S. 33) das Konzept des „Mathematics Learning Support System“ (MLSS). Demzufolge kann ein Kind Objekten, Begriffen oder Handlungen nicht unmittelbar einen mathematischen Sinn zuschreiben, sondern benötigt vielmehr ein kognitiv herausforderndes soziales Unterstützungssystem, das MLSS, in dem z. B. Fragen gestellt, Hypothesen aufgestellt und überprüft werden, für Ideen argumentiert wird und sich so schrittweise eine mathematische Sichtweise entwickeln kann (vgl. auch das Konzept des „Mathematics Acquisition Support System“ bei Tiedemann 2012). So lernen Kinder Mathematik in mathematikhaltigen Interaktionsprozessen mit fachlich in der Interaktion fortgeschrittenen Personen durch eine allmähliche Verschiebung des Autonomiegrades. Sie erhalten zunehmend die Möglichkeit eigenverantwortliche Beiträge in die mathematikhaltigen Interaktionsprozesse einzubringen, wodurch sie in Anlehnung an das Konzept des Mutterspracherwerbs nicht nur die notwendigen mathematischen Begriffe erlernen, sondern auch in die Art und Weise der mathematischen Kommunikation eingeführt werden (vgl. Jung 2019).

Diese beiden Modelle der sozial konstituierten Lernermöglichungsbedingungen werden in dem Begriff des „Argumentationsformats“ (Krummheuer und Brandt 2001, S. 20) zusammengeführt. Argumentationsformate sind als spezifische, argumentativ geprägte, lernförderliche Ausprägungen von Formaten - also Interaktionsmustern mit der Tendenz einer Rollenverschiebung der Autonomie hin zu dem in der Interaktion weniger fortgeschrittenen Individuen - zu verstehen. Folglich ist nach Krummheuer und Brandt (2001) die grundlegende soziale Bedingung für die Möglichkeit mathematischen Lernens die Partizipation an emergierenden Argumentationsformaten zu Aussagen über mathematische Inhalte, Begriffe oder Verfahren. Das schwierig zu fassende Zusammenspiel von individuellen und sozialen Konstituenten des Lernens lässt sich zusammenfassend wie folgt beschreiben: Das Individuum hat aufgrund der durch erfolgreiche Partizipation an kollektiven Argumentationen hervorgerufenen individuellen Neukonstruktionen und Modifikationen von Rahmungen wiederum eine gesteigerte Möglichkeit erfolgreich an zukünftig folgenden kollektiven Argumentationen zu partizipieren. Durch die Partizipation an kollektiven Argumentationen entsteht neben der Möglichkeit zum Erwerb von mathematischen Begriffen und Prozeduren im Sinne eines Lernens von Inhalten, zudem die Möglichkeit des über den Inhalten, liegenden“ Argumentierenlernens im Sinne einer Einführung in eine mathematische Argumentationskultur. Im Folgenden werden diese beiden Aspekte des Mathematiklernens aus interaktionistischer Perspektive genauer voneinander differenziert.

\subsection{Zwei Aspekte des Mathematiklernens: Der Erwerb von Begriffen und Prozeduren und die Entwicklung mathematischen Denkens}

Für eine spezifischere Ausdifferenzierung unseres Verständnisses von ,Mathematiklernen" greifen wir erneut auf die lerntheoretischen Ausführungen von Bruner (2002) zum frühen Mutterspracherwerb zurück. Bruner (2002, S. 8) betont, dass innerhalb des LASS Sprache von den Lernenden nicht nur im Sinne des Entschlüsselns eines Sprachcodes erworben wird, sondern zudem auch im umfänglicheren Sinne die Kultur und das ,Bewegen“ in dieser gelernt wird (siehe auch Hausen- 


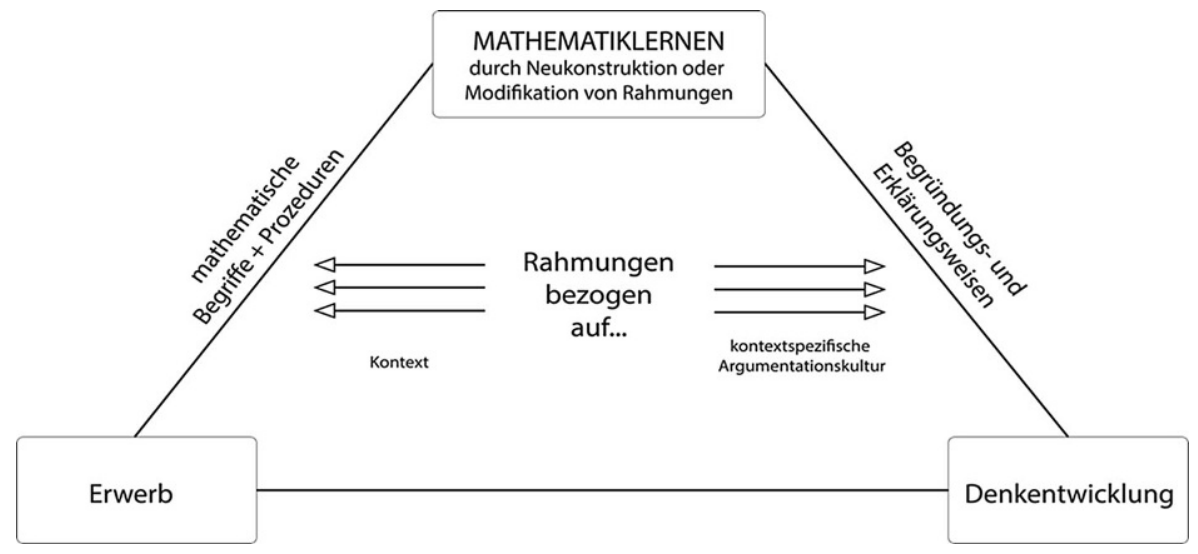

Abb. 1 Zwei Aspekte des Mathematiklernens. (Schütte und Krummheuer 2017, S. 878)

dorf und Quasthoff 1996; Krummheuer 2011). Überträgt man diesen Gedanken auf frühe mathematische Entwicklungsprozesse, so kann man analytisch zwischen dem Erwerb mathematischer Begriffe und Prozeduren im Sinne des Knackens eines mathematischen Codes und der Entwicklung mathematischen Denkens im Sinne einer Auseinandersetzung mit den $\mathrm{zu}$ diesen Inhaltsbereichen etablierten Begründungsund Erklärungsweisen gleichsam der mathematischen Rationalisierungspraxis ${ }^{4}$ differenzieren (siehe Abb. 1). Dieser zweite Aspekt des Mathematiklernens, hier als mathematische Denkentwicklung bezeichnet, lässt sich auch als ein Argumentierenlernen innerhalb des logisch-argumentativen Begriffs- und Strukturwerkes der Mathematik beschreiben.

Als Beispiel des Erwerbs mathematischer Begriffe und Prozeduren seien z.B. das Erlernen der Zahlwortreihe oder das Erlernen von Rechengesetzen der Grundrechenarten erwähnt. Mit der Genese einer Theorie mathematischer Denkentwicklung sollen über diesen reinen Erwerb von Begriffen und Prozeduren hinaus, die mit diesen mathematischen Inhalten verbundenen Argumentationsweisen einer je spezifischen Rationalisierungspraxis in den Blick genommen werden.

Bei dem Begriff mathematische Denkentwicklung denken wir hiernach an Aspekte der Interaktion, anhand derer sich rekonstruieren lässt, dass Individuen aufgrund ihrer Teilnahme an kollektiven Argumentationen veränderte oder neue argumentatorische Rahmungen zur Konstruktion ihrer individuellen Situationsdefinition hinzuziehen. Diese Neukonstruktion oder Weiterentwicklung der argumentatorischen Rahmungen führen dann zu der Möglichkeit einer autonomeren Partizipation an einer mathematisch elaborierteren Rationalisierungspraxis. Somit verwenden wir den Begriff Mathematiklernen, wenn wir den Prozess der kognitiven Konstruktion oder

\footnotetext{
4 Als Rationalisierungspraxis bezeichnen wir die empirisch zu rekonstruierenden Begründungs- und Erklärungsweisen einer bestimmten Gruppe von Menschen bezogen auf ein spezielles Gebiet, zum Beispiel die einer Klassengemeinschaft beim Mathematiklernen. Unter Argumentationskultur verstehen wir hingegen situationell übergreifende und kulturell entstandene Argumentationsweisen bezüglich eines speziellen Gebietes, in diesem Fall innerhalb der Wissenschaft der Mathematik.
} 
Weiterentwicklung einer inhaltlich-kontextuellen Rahmung und einer argumentativen-kontextspezifischen Rahmung beschreiben, die bei dem Individuum mit einer autonomeren Partizipation an den betreffenden mathematikspezifischen Interaktionen einhergeht.

\section{Eine theoretische und empirische Erweiterung der interaktionistischen Perspektive: Mathematische Denkentwicklung durch Partizipation an Diskursen}

Für eine longitudinale Perspektive mathematischer Denkentwicklung benötigen wir eine grundlegende Theorie, auf deren Basis auch längerfristige Prozesse der Konstruktion oder Modifikation von argumentatorischen Rahmungen von Kindern bei der Teilhabe an spezifischen mathematischen Diskursen beschrieben werden können. Es erscheint nötig, den in der Interaktionstheorie ausgebauten Ansatz zur Beschreibung von mathematischen Lernprozessen im Sinne einer lokalen Theorieentwicklung (Kelle 1994) entsprechend zu erweitern. Theoriegenese lässt sich nach Kelle (1994) nur sehr eingeschränkt durch die gängigen Schlussweisen der Induktion und Deduktion betreiben, sondern wird vor allem durch den von Peirce entwickelten Schlussmodus des hypothetischen Schließens umgesetzt (vgl. Hoopes 1991; siehe für eine vertiefte Auseinandersetzung zur Theorienentwicklung auch Reichertz 2013). Unter die Forschungslogik des hypothetischen Schließens fallen die von Peirce entwickelten Schlussmodi der qualitativen Induktion und Abduktion. Werden beim hypothetischen Schließen empirische Phänomene als Fälle von bekannten Regeln bzw. Theorien erklärt, fällt diese Art der Theorieentwicklung in den Bereich der qualitativen Induktion. Eine solche Form des Schließens ist häufig jedoch nicht möglich, wenn die bestehende Theorie Phänomene der Empirie nicht zu beschreiben oder erklären vermag bzw. widersprüchliche Interpretationen von beobachteten Fällen auftreten. In diesem Sinne stellt das ,Scheitern“ der qualitativen Induktion den Beginn von Theorieentwicklung dar. Es bedarf einer weiteren Schlussform, der Abduktion. Akzeptierte Annahmen werden in dieser zur Disposition gestellt und die durch das bis dato geltende theoretische Wissen abgesteckten Grenzen überschritten (vgl. Kelle 1994; Reichertz 2013). Durch ein unerwartetes Phänomen können die Forschenden angeregt sein, neue Regeln zu konstruieren, die dieses Phänomen zu erklären vermögen. Abduktives Schließen ermöglicht es, vorhandene theoretische Überlegungen in die Konstruktion von neuen abduktiv gewonnenen Theorieelementen zu integrieren. In der Peirce'schen Terminologie stellen abduktive Schlussformen den eigentlichen generativen Aspekt von Theorieentwicklung dar, bei dem Theorie aus dem Gegenstand selbst entwickelt wird.

Für unser Interesse an einer empirischen Rekonstruktion mathematischer Denkentwicklung von Kindern erscheint es uns - wie bereits ausgeführt - wesentlich, die Form des Argumentierens und den damit verbundenen Prozess des Argumentierenlernens deutlicher sichtbar werden zu lassen. Es soll somit nicht nur analysiert werden, wie und in welcher Art mathematische Begriffe oder Prozeduren in der Interaktion ausgehandelt werden, sondern auch mit welchen argumentativen und semantischen Strukturierungen sich die mathematische Themenentwicklung in den 
sequentiell ablaufenden kollektiven Argumentationen vollzieht. Es soll beispielweise unter anderem genauer betrachtet werden, wie die Objekte der Aushandlung innerhalb der analysierten Argumentationen bestimmt und thematisiert werden, wie der Argumentationsprozess in seinem zeitlich oder logischen Aufbau strukturiert ist, ob Begründungen der Beteiligten eher universelle oder partikuläre Gültigkeit besitzen und inwiefern handelnde Subjekte innerhalb der emergierenden mathematischen Gespräche eine Rolle spielen. Wir charakterisieren deshalb die emergierenden Aushandlungsprozesse als Diskurse mit spezifischen argumentativen und semantischen Strukturierungen, die wir dann als ,Diskursstil ' bezeichnen. Solche spezifischen Diskurse über mathematische Inhalte lassen sich als interaktive Realisierungen eines MLSS, verstehen, welches zwischen allen Beteiligten innerhalb einer oder mehrerer ,zusammenhängender' kollektiver Argumentationen erzeugt bzw. interaktiv ausgehandelt wird. Im Rahmen dieses Beitrages soll deshalb als erster Teil des Forschungsvorhabens folgende Frage beantwortet werden: Welche diskursiven Strukturen lassen sich beim frühen Mathematiklernen in kollektiven Argumentationen rekonstruieren?

Zur Beantwortung dieser Forschungsfrage wird als empirisches Datenmaterial auf Videoaufnahmen von Spiel- und Erkundungsumgebungen aus dem Projekt erStMaL (early Steps in Mathematics Learning) des Frankfurter IDeA-Zentrums ${ }^{5}$ zurückgegriffen (für weitere Informationen zum Projekt siehe Brandt et al. 2011). Im Rahmen der Longitudinalstudie bearbeiteten Kinder im Alter von drei bis neun Jahren in verschiedenen Gruppenzusammensetzungen (zwei bis vier Kinder) mit einer Projektmitarbeiterin oder einem Projektmitarbeiter mathematische Spiel- und Erkundungsumgebungen, die alle relevanten mathematischen Inhaltsbereiche der frühen mathematischen Bildung (für die Beschreibung der Inhaltsbereiche siehe Clements und Sarama 2007) berücksichtigten. Insgesamt sind in einem circa halbjährlichen Abstand sechs aufeinanderfolgende Erhebungsphasen durchgeführt worden. In jeder Erhebungsphase hat jedes Kind in der Regel an zwei unterschiedlichen Spiel- und Erkundungsumgebungen teilgenommen. Die Studie ist in der ersten Erhebungswelle mit 120 Kinder als Kerngruppe und circa 25 weiteren Kindern aus einer Ergänzungsgruppe gestartet.

Die Beschreibung der Spiel- und Erkundungsumgebungen liegen in der Form sogenannter „Didaktischen Design Patterns“ (Acar Bayraktar et al. 2011, S. 17; zum Begriff siehe auch Vogel und Wippermann 2005) vor. Diese gibt den Projektmitarbeiterinnen und Projektmitarbeitern einen Rahmen vor, wie in die Spielund Erkundungsumgebung eingeführt bzw. die Aushandlung der Kinder fortführend unterstützt werden kann und welche Materialien zur Verfügung stehen. Diese Hilfestellungen sollen jedoch die ergebnisoffene und interaktive Komponente der Bearbeitung der Lernumgebungen nicht einschränken. So sind in den Design Patterns keine Ziele formuliert, die die Beteiligten erreichen sollen. Ebenso wenig ist

\footnotetext{
${ }^{5}$ IDeA steht für Individual Development and Adaptive Education of Children at Risk und ist ein interdisziplinäres Forschungszentrum, das es sich zur Aufgabe gemacht hat, in unterschiedlichen Disziplinen Entwicklungsbedingungen von Kindern mit sogenannten Risikofaktoren zu erforschen und langfristig geeignete Fördermöglichkeiten für die Praxis zu entwickeln. Es wurde in einer Kooperation des Deutschen Instituts für Internationale Pädagogische Forschung, des Sigmund-Freud-Instituts und der Goethe-Universität Frankfurt eingerichtet.
} 
es angedacht einzugreifen, falls zwischen den Kindern Themen der Aushandlung virulent werden, die in den Design Pattern nicht bedacht worden sind.

Das methodische Vorgehen der Analyse der gewonnenen Daten lässt sich im Bereich interaktionistischer Arbeiten der Interpretativen Unterrichtsforschung der Mathematikdidaktik (vgl. Krummheuer und Brandt 2001; Krummheuer und Naujok 1999; Schütte 2009; Voigt 1995) verorten. Bei der Analyse der Interaktionen orientieren wir uns an einer rekonstruktiv-interpretativen Methodologie und an einem zentralen Element des Forschungsstils der Grounded Theory - dem methodischen Ansatz der komparativen Analyse (vgl. Bohnsack 2007; Strauss und Corbin 1996; zur Anwendung in der Mathematikdidaktik siehe auch Krummheuer und Brandt 2001; Schütte 2009). Innerhalb dieses komparativen Vorgehens wurden vier Kinder aus der Kerngruppe ausgewählt, von denen ein möglichst vollständiger Datensatz über alle Erhebungswellen vorliegt, wobei auf eine Gleichverteilung von Jungen und Mädchen sowie von Kindern mit und ohne Migrationshintergrund geachtet wurde. Die so aus dem gesamten Datenkorpus ausgewählten Episoden wurden mithilfe der Interaktionsanalyse (vgl. Krummheuer 2012; Krummheuer und Naujok 1999; Schütte et al. 2019) ausgewertet. Mit Blick auf die selbst nur bei diesen vier Kindern vorliegende umfassende Datenmenge - circa 40 Episoden - sind die vorliegenden Analysen als illustrierende Beispiele der sich im Prozess befindenden Analysen zu verstehen.

\section{Ergebnisse der empirischen Analysen: Rekonstruktion diskursiver Strukturen beim Mathematiklernen in frühen kollektiven Argumentationen}

Im folgenden Abschn. 4.1 stellen wir Einblicke in die Analysen zweier Diskurssequenzen einer Episode aus dem Datenmaterial des erStMaL-Projektes vor. Die Sequenzen dienen zur Illustration des Theoretisierungsprozesses und sollen bestmöglich die je spezifischen argumentativen und semantischen Strukturierungen der jeweiligen Diskurse am Beispiel verdeutlichen. Anzumerken ist, dass sich ein solcher Theoretisierungsprozess nur aus der Komparation von Analysen vieler Diskurse ergibt, wodurch eine Eins-zu-Eins-Deckung zwischen dem illustrierenden Beispiel und dem daraus gezogenen Theorieextrakt - den spezifischen argumentativen und semantischen Strukturierungen der Diskurse - nur im Ansatz gelingen kann. Im Anschluss daran fassen wir jeweils die theoretisch abgeleiteten und empirischen ausdifferenzierten Charakteristika der beiden Diskursstile zusammen bevor in Abschn. 4.2 das in der abduktiven Logik überraschende Phänomen eines in der Ausgangstheorie nicht bedachten Diskurses, der ,narratorische Diskurs', als Verbindungsraum der beiden Diskursstile beschrieben und ebenfalls mit einer Beispielsequenz illustriert wird. 


\subsection{Qualitative Induktion: Narrative und formale Diskurse}

\subsubsection{Rekonstruktion eines formalen Diskurses}

An der hier vorliegenden kombinatorischen Spiel- und Erkundungsumgebung nehmen vier Kinder - Marie (5;10), Chris $(5 ; 3)$, René $(5 ; 11)$ und Levent $(5 ; 10)$ - unter der Anleitung einer Projektmitarbeiterin teil. Zu Beginn der Episode sitzen die vier Kinder zusammen auf dem Fußboden. Vor ihnen liegen zwei Teppiche. Auf diesen befinden sich Papierfiguren in Form einer Hose, eines Pullovers und einer Mütze jeweils mehrfach in den Farben rot, gelb und blau.

\begin{tabular}{|c|c|c|c|}
\hline 01 & & Marie & $\begin{array}{l}\text { ähm da sind ganz viele ähm verschiedene ä h } \mathrm{m} \text { [zeigt auf die Papier- } \\
\text { figuren auf dem Teppich] ähm eine Mütze ist rot die passt zu einem } \\
\text { roten T-Shirt [legt roten Pullover mit roter Mütze zusammen] }\end{array}$ \\
\hline 02 & & $\mathrm{P}$ & mhm [zustimmend $]$ \\
\hline 03 & & Marie & $\begin{array}{l}\text { dann passt ähm die Hose zu zu dem roten T-Shirt [legt rote Hose } \\
\text { zu roter Mütze und rotem Pullover] < dann passt . die die Sachen } \\
\text { zusammen [ordnet blaue Hose, blauen Pullover, blaue Mütze von } \\
\text { unten nach oben an] }\end{array}$ \\
\hline 04 & $<$ & Chris & $\begin{array}{l}\text { [ordnet gelbe Hose, gelben Pullover, gelbe Mütze von unten nach oben } \\
\text { an] }\end{array}$ \\
\hline 05 & & Marie & dann muss noch die so zusammenl \\
\hline 06 & & $\mathrm{P}$ & achl \\
\hline 07 & & Marie & das sind alles Sachen die zusammenpassen\ \\
\hline 08 & & $\mathrm{P}$ & warum passen denn die Sachen besonders gut zusammen/ \\
\hline 09 & $<$ & Marie & ähm [zeigt nacheinander auf rote Hose, roten Pullover, rote Mütze] \\
\hline 10 & $<$ & Chris & weil die alle die gleiche Farbe haben\ \\
\hline
\end{tabular}

Ohne, dass ein Arbeitsauftrag an die Kinder gestellt wird, beginnt Marie die bunten Kleidungsstücke auf dem Teppich zu ordnen. Es scheint als verfolge sie ein für sie klar strukturiertes Vorgehen, indem sie zum einen die Kleidungsstücke anhand der Kategorie Farbe sortiert $<01,03>$ und zum anderen innerhalb einer Farbe die Kleidungsstücke in einer bestimmten Reihenfolge (Hose, Pullover, Mütze) anordnet $<03>$. Sie bildet somit Gruppierungen aus je einer Mütze, einem Pullover und einer Hose und folgt bei der Anordnung dieser Elemente der Logik eines angezogenen Menschen. Chris scheint Maries Ordnungskategorien direkt zu erkennen und ist in der Lage diese selbst unmittelbar anzuwenden $<04>$. Dies wird durch Chris Antwort „weil die alle die gleiche Farbe haben “ <10> auf die Frage der Projektmitarbeiterin bestätigt. Die beiden Kinder bringen so gleich zu Beginn der Situation ein Deutungsinterim hervor, das ihnen ermöglicht durch ihre gemeinsamen Handlungen und verbalsprachlichen Äußerungen ein kollektives Argument (zur Differenzierung von kollektiver Argumentation und der Erzeugung eines kollektiven Arguments siehe Jung 2019) zu entwickeln.

Betrachten wir die argumentative und semantische Strukturierung in dem hier emergierenden Diskurs, fällt Folgendes auf: Die entwickelten Ordnungen der Kinder werden anhand von äußeren Eigenschaften der Kleidungsstücke, anhand ihrer Form oder Farbe, vorgenommen. Der emergierende Aushandlungsprozess bezieht 
sich auf die Kleidungsstücke, ihre Eigenschaften und ihre Beziehungen untereinander. Begründungen innerhalb der kollektiven Argumente scheinen eine gewisse universelle Gültigkeit zu besitzen oder zumindest über die Situation und den Kontext hinauszuweisen. Es werden von den Kindern keine Bezüge zwischen der von ihnen entwickelten Ordnung der Kleidungsstücke und realen Erlebnissen hergestellt. Nur die Verwendung des Adverbs ,,dann“ in der Aufzählung Maries erinnert ein wenig an eine zeitlich-sequentielle Darstellung einer Erzählung. Die von den Kindern hervorgebrachten Permutationen entspringen einer für Marie und Chris konstruierten formalen Logik.

Wir bezeichnen einen solchen Diskurs im Weiteren als einen formalen Diskurs. Ein formaler Diskurs zeichnet sich nach unseren Analysen durch folgende argumentative und semantische Strukturierungen aus:

- Relationen zwischen Objekten und charakteristischen Eigenschaften der Objekte stehen im Fokus des Aushandlungsprozesses;

- der Diskurs ist inhaltlich-relational und damit auch logisch-sequentiell ${ }^{6}$ struktu- $^{-}$ riert (bspw.: wenn ..., dann ...);

- die charakteristischen Eigenschaften der Objekte werden durch eine formale Logik bestimmt;

- es werden universelle Begründungen verwendet oder entwickelt.

Zur Komplettierung für den gleich anstehenden Vergleich mit einem narrativen Diskurs könnte ergänzt werden, dass im formalen Diskurs keine alltäglichen Erfahrungshintergründe expliziert werden und es keine handelnden Subjekte gibt.

\subsubsection{Rekonstruktion eines narrativen Diskurses}

Die oben dargestellte Sequenz läuft weiter, indem Marie eine neue Idee einbringt „aber wenn wir die mischen wird es ganz bunt" und diese auch unmittelbar beginnt umzusetzen. Am Ende ihres zweiten Sortierprozesses sind die Kleidungsstücke so angeordnet, sodass in jedem Outfit jeweils die Mütze, der Pullover und die Hose eine andere Farbe besitzen. Marie beendet ihre Handlung mit den Worten , aber dafür ist das dann durcheinander". Sie benennt auf Nachfrage der Projektmitarbeiterin die entstandenen Farbanordnungen jedes Outfits.

In diesem Moment wird die Papierpuppe Kim von der Projektmitarbeiterin in die Interaktion eingebracht „das ist übrigens Kim und die möchte jetzt zu einer Geburtstagsfeier gehen und die hat wie ihr das hier schon hingelegt habt drei verschiedene Pullover ne/ in welchen Farben/".

\footnotetext{
6 Wir sprechen von „logisch-sequentiell“ um das Charakteristikum eines formalen Diskurses mit logischen Implikationen von dem Charakteristikum der zeitlichen Sequentialität einer Narration zu unterscheiden, wie z. B. bei der Aussage: ,Wenn die Quersumme einer natürlichen Zahl n durch 3 teilbar ist, dann ist auch n durch 3 teilbar“. Diese logisch-sequentielle Folgerung impliziert kein Vorher und Nachher, wie beispielsweise bei folgendem Satz: „Peter wacht auf, dann geht er ins Badezimmer und putzt sich dann seine Zähne.“, was einer zeitlich-sequentiellen Strukturierung eines narrativen Diskurses entspricht.
} 


\begin{tabular}{|c|c|c|c|}
\hline 23 & & Marie & $\begin{array}{l}\text { wir könnten sie ja auch wenn sie zu nem Mädchengeburtstag geht } \\
\text { könnte rot oder gelb passen [nimmt gelbe Hose, gelbe Mütze, gelben } \\
\text { Pullover] }\end{array}$ \\
\hline 24 & & $\mathrm{P}$ & warum passt das gut bei nem Mädchengeburtstag \\
\hline 25 & & Marie & weil Mädchen manchmal rot oder gelb sehr gerne mögen \\
\hline 26 & & $\mathrm{P}$ & $\begin{array}{l}\text { okay .. gut dann wenn sie dann zu nem Mädchengeburtstag geht was } \\
\text { müsste sie denn bei einem Jungengeburtstag anziehen Levent/ }\end{array}$ \\
\hline 27 & & Chris & blau \\
\hline 28 & & Levent & blau \\
\hline 29 & & $\mathrm{P}$ & warum/ \\
\hline 30 & & Marie & ich < weiß es \\
\hline 31 & $<$ & Chris & weil sies mögen \\
\hline 32 & & $\mathrm{P}$ & $\mathrm{ja} /$ \\
\hline 33 & & Marie & Jungs mögen gerne blau \\
\hline 34 & & $\mathrm{P}$ & okay \\
\hline 35 & & Marie & ich kenne ganz viele die gerne blau mögen \\
\hline 36 & & $\mathrm{P}$ & $\begin{array}{l}\text { ja und jetzt steht die Kim vor ihrem Kleiderschrank ja und sie hat ja } \\
\text { eben verschiedene Farben Pullover verschiedene Farben }<\text { an Hosen }\end{array}$ \\
\hline 37 & $<$ & Marie & aber erstmal muss man wissen wo sie hingeht/ \\
\hline 38 & & $\mathrm{P}$ & zum Geburtstag \\
\hline 39 & & Marie & ja aber welches Geburtstag das muss man wissen \\
\hline 40 & & $\mathrm{P}$ & okay \\
\hline 41 & & Marie & $\begin{array}{l}\text { sonst weiß man ja nicht was für eine Kleidung wenns zum Mädchen- } \\
\text { geburtstag haben wir ein Problem weil da zwei sind }\end{array}$ \\
\hline 42 & & $\mathrm{P}$ & okay \\
\hline 43 & & Marie & dann müssen wir dann muss dann muss das Mädchen entscheiden \\
\hline 44 & & $\mathrm{P}$ & $\begin{array}{l}\text { okay dann sagen wir mal sie geht jetzt zuerst zu nem Mädchengeburts- } \\
\text { tag }\end{array}$ \\
\hline 45 & & Marie & $\begin{array}{l}\text { gut okay jetzt müssen wir erstmal anfangen zu rätseln [hält Papier- } \\
\text { puppe in der Hand und stellt sie vertikal zu den roten und gelben } \\
\text { Kleidungsstücken] mh vielleicht ziehen wir ihr es einfach mal an und } \\
\text { schaun wie es ihr steht }<\text { [legt Papierpuppe wieder hin und legt gelben } \\
\text { Pullover und gelbe Hose auf sie] }\end{array}$ \\
\hline 46 & $<$ & Chris & [legt gelbe Mütze auf die Papierpuppe] \\
\hline 47 & & Marie & passt das oder nich/ \\
\hline 48 & & René & mhmh $[$ verneinend $]$ \\
\hline 49 & & Marie & gut [nimmt gelbe Kleidungsstücke von der Papierpuppe] \\
\hline 50 & & $\mathrm{P}$ & warum passt das nicht René/ \\
\hline 51 & & René & weil es nicht schön aussieht \\
\hline
\end{tabular}

Mit Bezug zu der von der Projektmitarbeiterin eingebrachten Geburtstagsgeschichte bringt Marie die Idee ein, dass für die Auswahl der Farbe des Outfits von Kim von Belang sein könnte, zu welchem Anlass das Outfit getragen werden soll <23>. Daraufhin entspinnt sich eine kollektive Argumentation, innerhalb derer die vier Kinder und die Projektmitarbeiterin ein Deutungsinterim aushandeln nach dem Kim, wenn sie zu einem Mädchengeburtstag gehen würde, andere Farben anziehen müsste, als wenn sie zu einem Jungengeburtstag ginge. Die Begründung für 
das richtige farbliche Outfit bezieht sich dabei auf die vermeintlichen Vorlieben von Mädchen und Jungen $<25,31,33>$. Hierdurch scheinen die unterschiedlichen Kombinationen nicht mehr auf der Basis charakteristischer Eigenschaften der Objekte, sondern aufgrund alltäglicher Erfahrungen der Kinder gefunden zu werden. Es treten soziale Erfahrungen als Begründung in den Vordergrund, wohingegen formale Begründungen eher in den Hintergrund treten. Diese angedachte fiktive Geschichte der Vorbereitung auf einen Geburtstag mit der Auswahl der angemessenen Kleidung bringt eine sequentiell strukturierte Narration eines Mädchens hervor, das vor seinem Kleiderschrank steht $<36>$, bevor es auf einem Mädchengeburtstag gehen möchte $<39-44>$ und deshalb ein Problem bekommt, weil für diesen Anlass mehrere mögliche Outfits zur Verfügung stehen $<41>$. Diese Narration wird in der folgenden Interaktion weiter entfaltet und das Handeln mit der Papierpuppe erhält kurzzeitig den Eindruck eines Puppentheaters <45>. Marie scheint vollends in der Geschichte ,aufzugehen“, sie begründet eine mögliche Auswahl der Kleidung damit, ob diese der Puppe stehen würde und schlägt zur Lösung das Anprobieren der Kleidung vor. René greift diese Rationalisierungspraxis auf und verwirft die Kombination aus gelben Kleidungsstücken mit der Begründung ,weil es nicht schön aussieht “ $<51>$. Es treten demnach ästhetische Kriterien in den Vordergrund der Aushandlung, die die Erstellung als für richtig zu beurteilende Kombinationen beeinflussen.

Betrachten wir den emergierenden Diskurs, ließe sich mutmaßen, dass es sich gar nicht mehr um einen mathematischen Diskurs halte, denn das vermeintlich klassisch typische Mathematische - das Formale und logisch Aufgebaute - scheint nicht mehr Kern der Aushandlung der Beteiligten zu sein. Vergewissert man sich jedoch, dass es sich bei den Beteiligten um Kinder handelt, die alle noch nicht das sechste Lebensjahr vollendet haben, lassen sich Aktivitäten des Klassifizierens, Seriierens und Strukturierens von alltäglichen Objekten im Bereich der frühen mathematischen Bildung als mathematische inhaltsübergreifende Basiskompetenzen (de Vries 2010) und spezifischer als Ordnung schaffende mathematische Denk- und Handlungsweisen (vgl. Rathgeb-Schnierer 2015) verstehen, die für den Aufbau einer mathematischen Grundbildung unerlässlich scheinen. Schauen wir uns somit die argumentative und semantische Strukturierung des emergierenden Diskurses an, scheint der Unterschied zum vorherigen formalen Diskurs zum einen in dem Einbezug alltäglicher Erfahrungen bzw. solcher aus der kindlichen Phantasiewelt zu liegen und zum anderen im Nachspielen/Nacherzählen einer, alltäglichen' Situation. Die Farbwahl für mögliche Kombinationen wird nicht mehr über formale Kriterien oder darüber welche Farbkombinationen theoretisch möglich wären bestimmt, sondern über subjektiv angenommene, sozial akzeptierte Farbauswahlen oder solche nach ästhetischen Gesichtspunkten. Die Kinder sind so an einer kollektiven Argumentation beteiligt, die in ihrer Gesamtheit eine fiktive Geschichte erzeugt oder die zumindest durch ihren erzählenden Charakter in der Form an eine erfahrungsbasierte Erzählung erinnert. Nicht die Relationen der Objekte zueinander mit den für sie charakteristischen Eigenschaften stehen im Vordergrund der Betrachtung, sondern das Soziale im Umgang mit diesen Objekten in alläglichen Situationen. Es emergiert ein Diskurs, den wir im Weiteren als einen narrativen Diskurs bezeichnen. Er zeichnet sich nach unseren Analysen durch folgende argumentative und semantische Strukturierungen aus: 
- Alltägliche Erfahrungen stehen im Fokus des Aushandlungsprozesses;

- der Diskurs ist zeitlich-sequentiell strukturiert (und dann ... und dann ...);

- es entspinnt sich eine sequentielle Dramaturgie einer Geschichte oder von Teilen einer Geschichte in erzählender Form;

- die charakteristischen Eigenschaften der Objekte werden durch den Kern der Geschichte bestimmt;

- es werden partikuläre, intuitive Begründungen verwendet oder entwickelt, die auf Erfahrungshintergründen im persönlichen Bezug zur emergierenden ,Geschichte beruhen;

- es gibt handelnden Subjekte.

Zur theoretischen Einordnung dieser zwei Arten von argumentativen und semantischen Strukturierungen der Diskurse als interaktiver Aspekt einer je spezifischen mathematischen Denkentwicklung lässt sich im Sinne der Qualitativen Induktion Bezug nehmen auf den narrativen Grundsatz des psycho-kulturellen Ansatzes zum Lernen und Denken von Bruner (1996). Bruner folgend lassen sich zwei verschiedene diametrale Modi des Denkens unterscheiden: narratives und logisch-wissenschaftliches Denken. Narratives Denken zeichnet sich dadurch aus, dass Menschen bestrebt sind in Form von Geschichten zu denken. Es geht um die Schaffung von Zusammenhängen, Sinn und einer Verortung von uns selbst in der Welt. Begründungen des narrativen Denkens zeichnen sich durch intuitive und partikulare Erklärungsmuster aus. Logisch-wissenschaftliches Denken hingegen fokussiert auf physikalische Objekte. Es geht um die Erfassung von Fakten, allgemeinen Regeln und Gesetzen der Welt. Begründungen des logisch-wissenschaftlichen Denkens zeichnen sich durch dekontextualisierte eher universell gültige Erklärungen aus. Beide Arten des Denkens liefern laut Bruner (1996) unterschiedliche Zugänge zur Welt und sind nicht ineinander überführbar.

\subsection{Abduktion: Narratorische Diskurse}

Im Gegensatz zur Aussage von Bruner (1996), der logisch-wissenschaftliches Denken und narratives Denken dualistisch trennt, finden wir im Sinne einer abduktiven Forschungslogik auch Diskurse, die unsere theoretische Einordnung mit Bezug zu Bruner (1996) in Frage stellen und sich nicht ohne Weiteres in eine der beiden Diskursformen einordnen lassen. So lässt sich eine weitere Diskursform rekonstruieren, die sich in den empirischen Analysen zwischen den narrativen und formalen Diskurs schiebt. An der im Folgenden dargestellten Spiel- und Erkundungsumgebung nehmen zwei Kinder, Marie (4;9) und René (4;10), teil. Während der Durchführung der Spiel- und Erkundungsumgebung aus dem Bereich der Mengenlehre und Logik sitzen die zwei Kinder mit der Projektmitarbeiterin an einem runden Tisch. Auf dem Tisch liegt ein grüner Teppich auf dem verschiedene große und kleine Marienkäferkarten ausgelegt sind. Die Marienkäferkarten sind unterschiedlich farbig (rot, grün oder gelb) und mit verschiedenen Markierungen (Punkten, Dreiecken oder Quadraten) versehen. Die beiden Kinder bekommen den Auftrag aus drei in der Mitte liegenden großen roten Marienkäfern einen nicht passenden Marienkäfer zu identifizieren. Es entspinnt sich folgender Aushandlungsprozess. 


\begin{tabular}{|c|c|c|c|}
\hline 750 & & $\mathrm{P}$ & $\begin{array}{l}\text { ich dreh's mal um dann kannst du's nämlich auch sehn\[dreht die } \\
\text { Karten in Maries Richtung] da passt auch einer nicht so richtig\ könnt } \\
\text { ihr den erkennen/ von den drei Großen/ [zeigt auf die drei großen, } \\
\text { roten Marienkäfer] }\end{array}$ \\
\hline 751 & $<$ & René & $\begin{array}{l}\text { [schnell] ja- [zeigt auf den großen Marienkäfer mit vielen kleinen Drei- } \\
\text { ecken, der rechts oben liegt] }\end{array}$ \\
\hline 752 & $<$ & $\mathrm{P}$ & $\begin{array}{l}\text { einer ist so ein bisschen/. [schaut auf die Karte auf der Renés Finger } \\
\text { liegt] mhml was denkst du Marie/ }\end{array}$ \\
\hline 753 & & Marie & $\begin{array}{l}\text { [zeigt mit dem Zeigefinger auf den großen, roten Marienkäfer mit den } \\
\text { großen Dreiecken] }\end{array}$ \\
\hline 754 & & René & $\begin{array}{l}\text { die hat so gezeigt\ [zeigt mit dem Mittelfinger ebenfalls auf den großen, } \\
\text { roten Marienkäfer mit den großen Dreiecken] }\end{array}$ \\
\hline 755 & $<$ & $\mathrm{P}$ & $\begin{array}{l}\text { das ist nicht schlimm } \backslash \text { [wendet sich an Marie] wieso meinst du dass } \\
\text { das- }\end{array}$ \\
\hline 756 & $<$ & Marie & $\begin{array}{l}\text { [legt die kleinen, gelben Marienkäfer, die sie in der Hand hatte, jeweils } \\
\text { auf eine Seite neben die beiden aufeinander gelegten großen, gelben } \\
\text { Marienkäfer] }\end{array}$ \\
\hline 757 & & $\mathrm{P}$ & $\begin{array}{l}\text { wieso denkst du das ist der/ [zeigt auf den rechts oben liegenden, gro- } \\
\text { ßen, roten Marienkäfer mit den kleinen Dreiecken] und wieso denkt } \\
\text { Marie das ist der\ [zeigt auf den Marienkäfer mit den großen Dreie- } \\
\text { cken] }\end{array}$ \\
\hline 758 & & Marie & $\begin{array}{l}\text { das ist der weil er so große Zacken hat } \backslash \text { [zeigt in die Richtung des gro- } \\
\text { ßen, roten Marienkäfers mit den großen Dreiecken] }\end{array}$ \\
\hline 759 & & $\mathrm{P}$ & ahal und du/ [schaut René an] \\
\hline 760 & & René & $\begin{array}{l}\mathrm{mhmh} \backslash[\text { schüttelt den Kopf und zeigt mit dem Finger auf den großen, } \\
\text { roten Marienkäfer mit den kleinen Dreiecken] }\end{array}$ \\
\hline 761 & $<$ & $\mathrm{P}$ & warum denkst du das ist der/ \\
\hline 762 & $<$ & Marie & [legt einen der kleinen, gelben Marienkäfer wieder auf die Kreisbahn] \\
\hline 763 & & René & weil er zu kleine hat $\backslash$ [kratzt sich am Kopf] \\
\hline 764 & & Marie & $\begin{array}{l}\text { [schiebt einzelne kleinen Marienkäfer auf der Kreisbahn wieder zusam- } \\
\text { men] }\end{array}$ \\
\hline 765 & & $\mathrm{P}$ & weil er was hat/ \\
\hline 766 & & René & $\begin{array}{l}\text { weil er zu kleine hat } \backslash \text { [zeigt mit dem Finger auf die kleinen Dreiecke } \\
\text { auf dem rechts oben liegenden, großen, roten Marienkäfer] }\end{array}$ \\
\hline 767 & & $\mathrm{P}$ & weil er zu kleinel. zu kleine als was $\$ \\
\hline 768 & & René & \begin{tabular}{l} 
zu kleine- vier- [schaut sich um, blickt zu kleinen Marienkäfern] hier so \\
Punkte\ [zeigt auf die kleinen Dreiecke des rechten, großen, roten Ma- \\
rienkäfers, nimmt eine kleine, rote Marienkäferkarte mit zwei kleinen \\
Punkten und zeigt sie $P$ ] wie die hier\ wie die kleinen\} \\
\hline 769 & & Marie & $\begin{array}{l}\text { ähm Renél [schaut sich interessiert Renés Karte mit dem kleinen Ma- } \\
\text { rienkäfer an, nimmt sie ebenfalls in die Hand, zusammen legen sie sie } \\
\text { wieder auf ihren Platz, zurück] }\end{array}$ \\
\hline
\end{tabular}
\end{tabular}




\begin{tabular}{|c|c|c|c|}
\hline 770 & & $\mathrm{P}$ & $\mathrm{hm} \backslash$ aber wir müssen uns jetzt einigen $\backslash$ also $\backslash$ einer $<$ passt nicht \\
\hline 771 & $<$ & René & $\begin{array}{l}\text { hey die sind- [streckt seine Hand in Richtung der großen, roten Ma- } \\
\text { rienkäfer] die zwei gehören nicht zu\[zeigt abwechselnd von einem } \\
\text { großen, roten Marienkäfer mit kleinen Dreiecken zum anderen] weil } \\
\text { die- gleichzeitig\ [zeigt immer wieder abwechselnd auf die Marienkä- } \\
\text { fer] }\end{array}$ \\
\hline 772 & & Marie & $\begin{array}{l}\text { [nimmt während des folgenden Dialogs zwei große, gelbe und einen } \\
\text { kleinen, gelben Marienkäfer hoch und ordnet sie auf dem Teppich neu } \\
\text { an] }\end{array}$ \\
\hline 773 & & $\mathrm{P}$ & die sind gleichzeitig\ \\
\hline 774 & & René & mhm [bejahend] \\
\hline 775 & & $\mathrm{P}$ & was meinst du denn damit $\backslash$ \\
\hline 776 & & René & $\begin{array}{l}\text { mh- des- der's der hat ein bisschen kleinere [zeigt auf den rechten, } \\
\text { großen, roten Marienkäfer mit den kleinen Dreiecken] noch als der/ } \\
\text { [zeigt auf den anderen großen, roten Marienkäfer mit den kleinen } \\
\text { Dreiecken] nämlich die wachsen } n \text { - und die wachs- und die sind schon } \\
\text { groß\ [zeigt auf den Marienkäfer mit den großen Dreiecken] }\end{array}$ \\
\hline 777 & & $\mathrm{P}$ & $\begin{array}{l}\text { aha } \backslash \text { also sind die doch größer\ [zeigt auf den großen, roten Marienkä- } \\
\text { fer mit den großen Dreiecken] }\end{array}$ \\
\hline 778 & & René & [schaut, nimmt eine Hand an den Mund, atmet aus, nickt langsam] \\
\hline
\end{tabular}

Die Projektmitarbeiterin legt ein Tripel von Marienkäfern in die Mitte des Tisches. Die drei Marienkäfer unterscheiden sich darin, dass bei zweien der Marienkäfer 19 kleine schwarze Dreiecke in gleicher Größe ungeordnet auf dem Rücken angeordnet sind und auf dem Rücken des dritten Marienkäfers sieben ungefähr dreimal so groBe schwarze Dreiecke in drei Reihen (3-3-1) angeordnet sind (zu diesem Transkript bereits veröffentlichte Analysen siehe in Krummheuer 2011, 2012; Hümmer et al. 2011). Die Sequenz beginnt mit der Frage der Projektmitarbeiterin: „,da passt auch einer nicht so richtig\. könnt ihr den erkennen von den drei Großen/“ $<750>$. René reagiert unmittelbar auf die Frage und zeigt auf einen der beiden Marienkäfer mit den kleinen Dreiecken $<751>$. Marie wählt den Marienkäfer mit den großen Dreiecken $<753$. Nach der Aufforderung der Projektmitarbeiterin < 757> begründet Marie ihre Auswahl wie folgt: ,das ist der weil er so große Zacken hatl“ $<758>$. Diese Aussage lässt die Deutung zu, dass die Dreiecke auf den Rücken der Marienkäfer Marie als Kriterium für ihre Entscheidung der Auswahl dienen. Damit scheint sie eine Begründung für die vordergründige und gegebenenfalls intendierte Lösung der Projektmitarbeiterin, dass der eine Marienkäfer mit den sieben großen Dreiecken aufgrund seiner Andersartigkeit zu den zwei anderen Marienkäfern nicht passen würde, hervorzubringen. Für René scheint diese Auswahl trotz der Begründung von Marie nicht passend $\langle 760\rangle$. Auf die wiederholte Nachfrage der Projektmitarbeiterin $<761>$ erklärt er seine Auswahl mit ,weil er zu kleine hatl“ $<763>$. Auf was sich seine Wortwahl „kleine“ bezieht lässt sich an dieser Stelle nicht zweifelfrei belegen. Es scheint so, als wenn er die sprachliche Struktur der Aussage von Marie aufgreift, als entscheidendes Merkmal jedoch nicht auf die ,großen Zacken', sondern auf die ,kleinen (Zacken) ' als Stützung seiner abweichenden Auswahl verweist. Die Projektmitarbeiterin fragt in $<765>$ nach einer weiteren Begründung, vermutlich da nun zwei divergierende Lösungen nebeneinanderstehen. René wiederholt 
seine Begründung in sprachlich identischer Form und zeigt dabei als Untermauerung auf die kleinen Dreiecke auf dem von ihm ausgewählten Marienkäfer $<766>$. Die Projektmitarbeiterin fragt mit ,zu kleine als wasl“ $<767>$ erneut nach. René antwortet ,zu kleine- vier- hier so Punktel wie die hier\ wie die kleinenl“ < $<68>$ und zeigt dabei zuerst auf die kleinen Dreiecke des großen Marienkäfers und dann auf die kleinen Marienkäferkarten als Referenzgruppe am Rand des Teppichs, die anscheinend einen Hinweis dafür liefern, dass die beiden Marienkäfer mit den 19 kleinen Dreiecken nicht passen. Die Projektmitarbeiterin gibt nun vor, dass eine Einigung notwendig sei. Währenddessen scheint René aufzufallen, dass es zwei große Marienkäfer mit kleinen Dreiecken gibt. Er benennt beide großen roten Marienkäfer mit kleinen Dreiecken als nicht passend $<771>$, begründet dies mit ,weil diegleichzeitig|“ $<771>$ sind und visualisiert seine Begründung mit einer Zeigegeste, welche zwischen den beiden großen roten Marienkäfern mit den kleinen Dreiecken hin und her wechselt. Diese ,Entdeckung ' des zweiten nicht passenden Marienkäfers lässt vermuten, dass René seine Auswahl des nicht passenden Marienkäfers zuvor nicht aufgrund des Abgleichs der drei großen Marienkäfer untereinander vorgenommen hatte, sondern in der ,Natur' des ausgewählten Marienkäfers eine Ursache zu liegen scheint, weswegen dieser nicht passen kann. Anscheinend liegt den unterschiedlichen Lösungen eine Rahmungsdifferenz zugrunde. Während Marie den einen Marienkäfer als unpassend auswählt, der aufgrund seiner großen Zacken nur einmal in der Dreiergruppe der Marienkäfer vorkommt, scheint René die Auswahl des unpassenden Marienkäfers vor einem anderen, hier nicht ohne weiteres rekonstruierbaren Hintergrund zu rahmen. Renés Erklärung „mh- des- der's der hat ein bisschen kleinere noch als der/ nämlich die wachsen n- und die wachs- und die sind schon groß\“<776> deutet daraufhin, dass er in den zwei von ihm ausgewählten und als gleichzeitig bezeichneten Marienkäferkarten mit je 19 Markierungen noch eine kleine Differenz in der Größe der Dreiecke festzustellen scheint. Dies könnte darauf zurückzuführen sein, dass die große Anzahl der Markierungen beider Marienkäfer einen Größenvergleich erschwert, da diese nicht systematisch auf dem Rücken der Marienkäfer angeordnet sind. Trotzdem scheint René beide in die gemeinsame Kategorie ,Marienkäfer, die noch wachsen' einzuteilen und damit von ,denen, die schon groß sind“ zu unterscheiden. Es gibt für ihn demnach die Unterscheidungskategorien ,klein` und ,groß` auf zwei Ebenen - einmal auf der Ebene der Marienkäfergröße und einmal auf der Ebene der Größe der Markierungen auf den Marienkäfern. Unter die Kategorie kleiner Marienkäfer fallen Renés Logik folgend alle Marienkäfer mit kleinen Markierungen. Diese Marienkäfer wachsen noch. Die großen ausgewachsenen oder erwachsenen Marienkäfer haben dieser Logik folgend große Markierungen auf dem Rücken. Genau wie Marie begründet René also die Differenz der drei großen Marienkäferkarten anhand der Größe ihrer Dreiecke auf dem Rücken. Im Unterschied zu Marie verbleibt er allerdings nicht bei diesem formal festgestellten Unterschied, sondern verbindet diese Einsicht vermutlich mit einer aus dem Alltag bekannten Gegebenheit. Renés ,gleichzeitig ' lässt sich somit auf zwei unterschiedliche Weisen deuten. Zum einen könnte damit zum Ausdruck kommen, dass die beiden großen Marienkäfer mit kleinen Markierungen die von ihm entworfene ,Ordnung ‘ verletzen, da ein Marienkäfer nicht groß sein kann und gleichzeitig kleine Markierungen auf dem Rücken haben kann. Zum anderen wäre 
es aber auch denkbar, dass beide Marienkäfer gleich sind in Bezug auf die Zeit also in Bezug auf das Alter. Sie haben beide die gleiche Anzahl von Dreiecken in gleicher Größe auf dem Rücken und sind damit gleich alt. Entscheidend dabei ist, dass die Logik beider möglicher Deutungen der Aussage von René ,weil diegleichzeitig|“ $<771>$ sich in einer implizit verborgenen Geschichte finden lässt in der Narration des Heranwachsens von Lebewesen bzw. Tier- oder Menschenkindern zu ,Tier- oder Menschenerwachsenen“. Die erfahrungsbasierte zwingende Sequentialität der Geschichte des (Heran-)Wachsens bestimmt hiernach die logische Ordnung, die René für die Marienkäfer wählt. Diese entspringt nicht rein formalen Kriterien einer inhaltlich-logischen Ordnung, sondern findet ihre Begründung in der zeitlich-sequentiellen Ordnung der Marienkäfer in der Geschichte. Große Marienkäfer haben große Markierungen auf ihren Rücken - so wie Erwachsene auch große Hände haben und damit die Fähigkeit besitzen große Rucksäcke tragen zu können und kleine Marienkäfer haben kleine Markierungen - so wie Kinder auch nur kleine Hände haben oder kleine Rücksäcke tragen können.

Fasst man die Analyse im Hinblick auf den emergierenden Diskursstil zusammen, lässt sich festhalten, dass während Marie zunächst im Sinne eines formalen Diskursstils eine in der Relation der betrachteten Objekte liegende formal logische Begründung für ihren Lösungsvorschlag angibt, René für die Begründung seines Lösungsvorschlages auf lebensnahe Alltagserfahrungen des (Heran-)Wachsens von Kindern zu Erwachsenen zurückgreift. Diese Erfahrungen werden in der Sequenz jedoch von ihm nicht in erzählender Form eingebracht, sondern entspringen nur noch implizit dem Plot bzw. Faktum einer erlebten Geschichte. So greift René auf die Form der Formulierung von Marie zurück, die mit diesen ihre formale Begründungslogik aufbaut. Hierdurch ergibt sich die Logik der Zuordnung von René zwar aus der Sequentialität der Geschichte, diese Sequentialität bzw. die Momente ihrer Hervorbringung, findet sich aber nicht mehr in ihrer Abfolge innerhalb des vorliegenden Diskurses. In diesem Sinne lässt sich der hier emergierende Diskurs nicht eindeutig einem der beiden Diskurse, narrativ oder formal, zuordnen. Von den Beteiligten wird keine Geschichte generiert, sondern innerhalb des Gespräches werden Aspekte einer fiktiven oder real erlebten Geschichte für Begründungen herangezogen, die aber formale Züge tragen. Narrationstheoretisch könnte man also davon sprechen, dass der Plot einer Geschichte thematisiert wird: es wird gleichsam noch in der ,Sprache“ der Geschichte über die Beziehungen zwischen den Subjekten und den Handlungsabläufen der Geschichte des (Heran-)Wachsens gesprochen, wodurch formale Aspekte thematisiert werden, die jedoch ,noch' an den Kontext einer zugrundeliegenden Geschichte gebunden sind.

Ein Diskursstil, der sich zwar implizit auf eine Geschichte bezieht, diese aber nicht explizit ausführt und so auch Aspekte eines formalen Diskurses beinhaltet, weil der Plot der Geschichte zu bestimmenden formalen Relationen der Objekte untereinander führt, ließe sich als quasi-narrativer bzw. quasi-formaler Diskurs bezeichnen. Es zeigt sich auf empirischer Ebene die Existenz eines in Veränderung befindlichen und somit nicht eindeutig zu typisierenden Diskurshybrides, den wir im 
Weiteren als narratorischen Diskurs ${ }^{7}$ bezeichnen werden. Ein narratorischer Diskurs zeichnet sich nach unseren Analysen durch folgende argumentativen und semantischen Strukturierungen aus:

- Beteiligte greifen weiterhin auf selbst erlebte Geschichten zurück - im Fokus des Aushandlungsprozesses stehen die Objekte und die mit ihnen verbundenen Erfahrungen;

- die Erfahrungen und charakteristischen Eigenschaften der Objekte werden nicht mehr in erzählender Form thematisiert;

- der Diskurs ist unspezifisch teils inhaltlich-relational bzw. logisch-sequentiell teils zeitlich-sequentiell strukturiert;

- die Bedeutung der Sequentialität des Erlebten wird in Momenten der inhaltlichrelationalen bzw. logisch-sequentiellen Struktur aufgebrochen;

- es werden partikuläre, intuitive Begründungen und universelle Begründungen verwendet oder entwickelt.

\section{Ausblick und lerntheoretische Einordnung: Lernen an der Schnittstelle der Diskurse}

In dieser und in weiteren Rekonstruktionen von Interaktionsprozessen aus den Episoden der Spiel- und Erkundungsumgebungen zeigt sich, dass sich rein narrative und rein formale Diskurse als theoretische Endpunkte eines Kontinuums von LehrLern-Arrangements verstehen lassen aber in empirischen Situationen nicht zwingend trennscharf auftreten. Die rekonstruierten mathematischen Diskurse in den analysierten Situationen bewegen sich vielfach zwischen beiden Diskursstilen hin und her und es emergieren auch narratorische Diskurse. Der in der folgenden Grafik abgebildete Torus soll das Ineinanderübergehen der drei Diskursstile formal, narrativ und narratorisch darstellen (siehe Abb. 2).

Die Rekonstruktion unterschiedlicher Diskursstile stellt einen wichtigen Schritt auf dem Weg der Genese einer Theorie mathematischer Denkentwicklung aus interaktionistischer Perspektive dar. Die Beantwortung der fokussierten Forschungsfrage schafft im Sinne einer zunehmenden empirisch gegründeten Theorieentwicklung die Grundlage dafür, in folgenden Analysen systematisch die Entwicklung mathematisch-argumentativer Fähigkeiten von Kindern longitudinal beschreiben zu können.

Abb. 2 Die drei Diskursstile: narrativ, narratorisch und formal

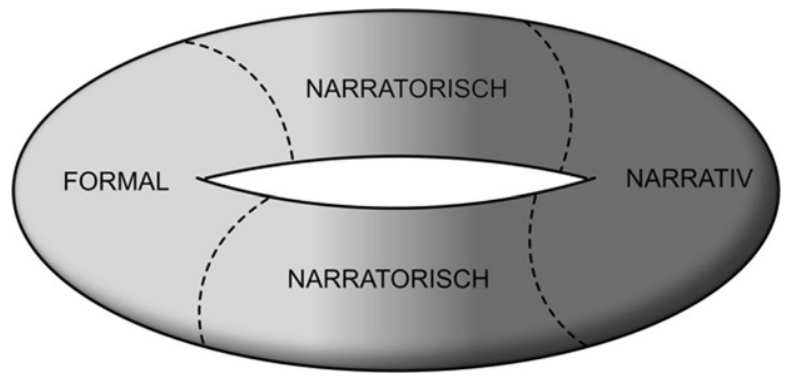

\footnotetext{
7 Der Begriff „,narratorisch“ wird hier in seiner Bedeutung als „eine Geschichte/Erzählung betreffend“
} verwendet. 
Für weitere Forschungsschritte stellt sich die Frage, welche Formen von unterschiedlichen individuellen Entwicklungslogiken mathematischer Denkentwicklung, angezeigt durch eine sich verändernde Partizipation an mathematischen Diskursen, sich bei Kindern im Vorschul- bis Grundschulalter rekonstruieren lassen. Longitudinale komparative Analysen von Maries Partizipation an unterschiedlichen mathematischen Diskursen geben bspw. Anlass zu der Vermutung, dass eine zunächst naheliegend scheinende Entwicklungslogik, einer zunehmend autonomeren Partizipation an narrativen Diskursen hinzu einer verstärkten Partizipation an formalen Diskursen, nicht der einzige Weg einer fortschreitenden mathematischen Denkentwicklung von Kindern zu sein scheint. Um diese weiterführenden Fragen zu beantworten, bedarf es eines modifizierten theoretischen Konstruktes, das es ermöglicht, die longitudinalen Veränderungen der Partizipation der Kinder beschreiben zu können und aus dieser den Verlauf ihrer mathematische Denkentwicklung zu rekonstruieren. Erste Ansätze könnte das bereits hierzu entwickelte Konzept der interaktionalen Nische mathematischer Denkentwicklung bieten (vgl. Krummheuer 2011).

Ohne diese Überlegungen zur Entwicklung einer Theorie der mathematischen Denkentwicklung im Detail weiterzuführen, lässt sich anhand der bereits erfolgten Analysen der zugrundeliegenden Untersuchung eine vorläufige lerntheoretische Einordnung vornehmen. Vergleicht man die ersten beiden analysierten Sequenzen aus der kombinatorischen Spiel- und Erkundungsumgebung, fallen zwei Aspekte auf. An dem emergierenden formalen Diskurs sind lediglich zwei Kinder - Marie und Chris aktiv beteiligt, wohingegen an dem emergierenden narrativen Diskurs alle Kinder aktiv partizipieren. Diese Beobachtung hebt illustrierend für bereits weitere von uns durchgeführte Analysen die Bedeutung narrativer Diskurse für das gemeinsame Lernen aller hervor. Narrative Diskurse sind, wie das obige Beispiel zeigt, mathematische Diskurse mit einer für viele Kinder niedrigen Zugangsschwelle, in denen, wenn auch mitunter verborgen, Aushandlungen über mathematische Inhalte oder Probleme stattfinden (siehe hierzu auch die Ausführungen zum impliziten Lernen bei Vogler 2020). So finden sich aktive Beiträge von René und Levent ausschließlich im narrativen Diskurs. Schaut man auf den narrativen Diskurs, fällt allerdings auch auf, dass das am autonomsten handelnde Kind, Marie, zwar sehr aktiv partizipiert, jedoch zunehmend in die Geschichte der Geburtstagsfeier, abtaucht ‘ und ihre Beiträge immer weniger einer ,formalen' Logik zu folgen scheinen. Aufgrund des Verweises der Projektmitarbeiterin auf den für Kinder alltäglichen Erfahrungshintergrund der Geburtstagsfeier ist Maries ,Abtauchen“ in diese Geschichte an dieser Stelle erzähllogisch nicht verwunderlich. Maries Anteil an der Hervorbringung des formalen Diskurses lässt jedoch vermuten, dass sie zumindest gewisse Fähigkeiten besitzt, formale Strukturen zu nutzen, um die sie umgebende Welt damit zu beschreiben. Der didaktische Ansatz, die Kinder mit einer alltagsnahen Geschichte behutsam in die Welt der Mathematik zu führen, scheint an dieser Stelle für Marie die Einnahme beziehungsweise Beibehaltung dieser logisch-mathematischen Perspektive zu erschweren. Dabei gehen wir nicht davon aus, dass Aushandlungen in formalen Diskursen das Ziel mathematischer Lehr-Lern-Arrangements sind. Für das Mathematiklernen in Gruppen mit vielfältigen Lernenden sollten auch vielfältige Zugänge geschaffen werden, die sowohl die Fähigkeiten von Kindern wie Marie als auch die Fähigkeiten von Kindern wie René und Levent berücksichtigen. Für einige 
Kinder wird ein narrativer Zugang jedoch gegebenenfalls zu einer Verschleierung des Zugangs zur logisch-argumentativen Struktur der Mathematik führen, wodurch die Möglichkeit eingeschränkt werden könnte, ihre vorhandenen formalen abstrakten Fähigkeiten weiterzuentwickeln. Dies ist aber auch nicht als Appell zu verstehen, Realitätsbezüge aus mathematischen Lehr-Lern-Arrangements zu verbannen. Für viele Kinder stellt ein realitätsnaher Zugang zur Mathematik mit der Möglichkeit des Anknüpfens an alltagsnahe Erfahrungen sicherlich durch die verstärkte Möglichkeit einer aktiven Mitgestaltung von narrativen mathematischen Diskursen einen guten Zugang zur Mathematik dar. Beiden Diskursstilen kommt in Bezug auf die spätere kindliche Entwicklung eventuell eine unterschiedliche aber nicht unbedingt weniger wichtige Funktion beim Mathematiklernen zu. Unsere Analysen zeigen auch auf, dass auf unterschiedliche Art und Weise innerhalb einer Spiel- und Erkundungsumgebung von einer zur anderen Diskursform gewechselt wird. Vielfach entstand der Wechsel plötzlich und folgte einer Initiierung der Projektmitarbeiterin, wenn sie bspw. die ersten Zugänge zur Aufgabe der Kinder unterbindet und eine Geschichte zur Erklärung der Aufgabenstellung einbringt. Uns erscheinen solche abrupten Wechsel wenig lernförderlich, da Lernende hierdurch kaum die Möglichkeit erhalten, ihre Fähigkeit an der Schnittstelle zwischen narrativem und formalem Diskurs zu partizipieren, aufzubauen.

Dies leitet zur Betrachtung des narratorischen Diskurses über. Für das Ziel einer mathematischen Grundbildung erscheint es sinnvoll, dass möglichst viele Kinder die Möglichkeit bekommen Kompetenzen im Mathematikunterricht zu erwerben, um zu lernen, erfolgreich an narrativen und formalen Diskursen zu partizipieren (zu informalen und formalen mathematischen Diskursen vgl. den Ansatz von Barwell 2016). Betrachtet man den analysierten narratorischen Diskurs fällt auf, dass sowohl Marie mit ihren Fähigkeiten an formalen Diskursen aktiv zu partizipieren als auch René, der eher an narrativen Diskursen aktiv zu partizipieren scheint, am narratorischen Diskurs partizipieren. Insofern könnte man vermuten, dass die lerntheoretische Bedeutung des narratorischen Diskurses vordergründig in der Möglichkeit zur gemeinsamen Teilhabe von unterschiedlichen Kindern an einem mathematischen Diskurs liegen könnte. Betrachten wir den emergierenden narratorischen Diskurs genauer, scheint es jedoch für alle Beteiligten eine Herausforderung zu sein, ein gemeinsames Deutungsinterim an der Schnittstelle von aus alltäglichen Erfahrungen entspringenden Begründungen und formal logischen Begründungen zu erzielen. Diese Beobachtung steht der Annahme einer für alle Lernenden erleichterten Möglichkeit zur Partizipation entgegen. Wir messen dem narratorischen Diskurs für die mathematische Denkentwicklung trotzdem oder gerade deshalb eine fundamentale entwicklungstheoretische Funktion zu, die sich - zugegebener Weise - erst in der weiteren longitudinalen Betrachtung bewähren muss. Ein Spezifikum optimierter früher mathematischer Aushandlungsprozesse sehen wir in der Hybridfunktion des narratorischen Diskurses also an der Schnittstelle von real Erlebbaren und so im Kontext Beschreibbaren und der Beschreibung und Aushandlung des von diesen Kontexten Gelöstem. Ein narratorischer Diskurs ist mit dieser Betrachtung eventuell gerade nicht zugangsfreier, sondern setzt erhöhte Anforderungen an Kinder. Er bietet hierdurch aber auch eine besondere Möglichkeit für die Entwicklung von spezifischen argumentativen Fähigkeiten - also die Möglichkeit Modifikationen und 
Neukonstruktionen von argumentatorischen Rahmungen aufgrund von emergierenden und auszuhandelnden Rahmungsdifferenzen zwischen den Beteiligten im gröBeren Stil zu evozieren als dies im narrativen oder formalen Diskurs der Fall zu sein scheint. Die interaktionalen Bedingungen für die Möglichkeit einer mathematischen Denkentwicklung sind so vor allem im narratorischen Diskurs gegeben. Dabei sehen wir durch die Teilhabe der Kinder an narratorischen Diskursen nicht monolinear eine Entwicklungsbewegung von narrativ $\mathrm{zu}$ formal sondern bi-modal sich erweiternde Partizipationschancen von Kindern für beide Diskurse.

\section{Transkriptionskonventionen}

\begin{tabular}{ll}
\hline [steht auf] & Handlung, Gesichtsausdrücke, Gesten \\
a 1 s o & Langsam und gedehnt gesprochen \\
/ & Stimmhebung \\
I & Stimmsenkung \\
. / . / ... & Sprechpausen für eins bis drei Sekunden \\
$<$ & Gleichzeitiges/überschneidendes Sprechen \\
P & Projektmitarbeiterin oder Projektmitarbeiter \\
\hline
\end{tabular}

Förderung Das Projekt erStMaL wurde gefördert durch die LOEWE-Initiative der Hessischen Landesregierung.

Funding Open Access funding enabled and organized by Projekt DEAL.

Open Access Dieser Artikel wird unter der Creative Commons Namensnennung 4.0 International Lizenz veröffentlicht, welche die Nutzung, Vervielfältigung, Bearbeitung, Verbreitung und Wiedergabe in jeglichem Medium und Format erlaubt, sofern Sie den/die ursprünglichen Autor(en) und die Quelle ordnungsgemäß nennen, einen Link zur Creative Commons Lizenz beifügen und angeben, ob Änderungen vorgenommen wurden.

Die in diesem Artikel enthaltenen Bilder und sonstiges Drittmaterial unterliegen ebenfalls der genannten Creative Commons Lizenz, sofern sich aus der Abbildungslegende nichts anderes ergibt. Sofern das betreffende Material nicht unter der genannten Creative Commons Lizenz steht und die betreffende Handlung nicht nach gesetzlichen Vorschriften erlaubt ist, ist für die oben aufgeführten Weiterverwendungen des Materials die Einwilligung des jeweiligen Rechteinhabers einzuholen.

Weitere Details zur Lizenz entnehmen Sie bitte der Lizenzinformation auf http://creativecommons.org/ licenses/by/4.0/deed.de.

\section{Literatur}

Acar Bayraktar, E., Hümmer, A.-M., Huth, M., Münz, M., \& Reimann, M. (2011). Forschungsmethodischer Rahmen der Projekte erStMaL und MaKreKi. In B. Brandt, R. Vogel \& G. Krummheuer (Hrsg.), Die Projekte erStMaL und MaKreKi. Mathematikdidaktische Forschung am „Center for Individual Development and Adaptive Education" (S. 11-24). Münster: Waxmann.

Barwell, R. (2016). Formal and informal mathematical discourses: Bakhtin and Vygotsky, dialogue and dialectic. Educational Studies in Mathematics, 92, 331-345.

Blumer, H. (2013). Der methodologische Standpunkt des Symbolischen Interaktionismus. In H. Bude \& M. Dellwing (Hrsg.), Symbolischer Interaktionismus. Aufsätze zu einer Wissenschaft der Interpretation (S. 63-140). Berlin: Suhrkamp. 
Bohnsack, R. (2007). Rekonstruktive Sozialforschung - Einführung in qualitative Methoden. Opladen: Barbara Budrich.

Brandt, B. (2004). Kinder als Lernende - Partizipationsspielräume und -profile im Klassenzimmer. Frankfurt: Peter Lang.

Brandt, B., Vogel, R., \& Krummheuer, G. (2011). Mathematikdidaktische Forschung am „Center for Individual Development and Adaptive Education". Grundlagen und erste Ergebnisse der Projekte erStMaL und MaKreKi. Bd. 1. Münster: Waxmann.

Bruner, J. (1996). The culture of education. Cambridge: Harvard University Press.

Bruner, J. (2002). Wie das Kind sprechen lernt. Bern: Huber.

Clements, D. H., \& Sarama, J. (2007). Early childhood mathematics learning. In F. K. Lester (Hrsg.), Second handbook of research on mathematics teaching and learning (S. 461-555). New York: Information Age.

Cobb, P., \& Bauersfeld, H. (1995). Introduction: The coordination of psychological and sociological perspectives in mathematics education. In P. Cobb \& H. Bauersfeld (Hrsg.), The emergence of mathematical meaning. Interaction in classroom cultures (S. 1-16). Hillsdale: Lawrence Erlbaum.

De Vries, C. (2010). Diagnostik und Förderung mathematischer Basiskompetenzen im Förderschwerpunkt geistige Entwicklung. Universität Oldenburg.

Doise, W., Mugny, G., \& Perret-Clerment, A.-N. (1975). Social interaction and the development of cognitive operations. European Journal of Social Psychology, 5(3), 367-383.

Ehm, J.-H., Lonnemann, J., \& Hasselhorn, M. (2017). Wie Kinder zwischen vier und acht Jahren lernen: Psychologische Erkenntnisse und Konsequenzen für die Praxis. Stuttgart: Kohlhammer.

Fetzer, M. (2007). Interaktion am Werk. Eine Interaktionstheorie fachlichen Lernens, entwickelt am Beispiel von Schreibanlässen im Mathematikunterricht der Grundschule. Bad Heilbrunn: Klinkhardt.

Fuson, K. C. (1988). Childrens' counting and concpts of number. New York: Springer.

Goffman (1959). The presentation of self in everyday life. New York: Anchor.

Hausendorf, H., \& Quasthoff, U. (1996). Sprachentwicklung und Interaktion: Eine linguistische Studie zum Erwerb von Diskursfähigkeiten bei Kindern. Wiesbaden: Westdeutscher Verlag.

Hoopes, J. (1991). Peirce on signs: writings on semiotic. Chapel Hill: UNC Press.

Hümmer, A., Münz, M., Müller Kirchof, M., Krummheuer, G., Leuzinger-Bohleber, M., \& Vogel, R. (2011). Erste Analysen zum Zusammenhang von mathematischer Kreativität und kindlicher Bindung. Ein interdisziplinärer Ansatz zur Untersuchung der Entwicklung mathematischer Kreativität bei sogenannten Risikokindern. In B. Brandt, R. Vogel \& G. Krummheuer (Hrsg.), Die Projekte erStMaL und MaKreKi. Mathematikdidaktische Forschung am „Center for Individual Development and Adaptive Education" (S. 175-196). Münster: Waxmann.

Jung, J. (2019). Möglichkeiten des gemeinsamen Lernens im inklusiven Mathematikunterricht - Eine interaktionistische Perspektive. In B. Brandt \& K. Tiedemann (Hrsg.), Mathematiklernen aus interpretativer Perspektive I-Aktuelle Themen, Arbeiten und Fragen (S. 103-126). Münster: Waxmann.

Jung, J., \& Schütte, M. (2018). An interactionist perspective on mathematics learning: conditions of learning opportunities in mixed-ability groups within linguistic negotiation processes. ZDM Mathematics Education, 50(6), 1098-1099.

Kelle, U. (1994). Empirisch begründete Theoriebildung. Zur Logik und Methodologie interpretativer Sozialforschung. Weinheim: Deutscher Studien Verlag.

Keller, R. (2012). Das interpretative Paradigma. Eine Einführung. Wiesbaden: Springer.

Krummheuer, G. (1992). Lernen mit „Format“. Elemente einer interaktionistischen Lerntheorie. Diskutiert an Beispielen mathematischen Unterrichts. Weinheim: Deutscher Studien Verlag.

Krummheuer, G. (1997). Zum Begriff der Argumentation im Rahmen einer Interaktionstheorie des Lernens und Lehrens von Mathematik. Zentralblatt für Didaktik der Mathematik, 29(1), 1-10.

Krummheuer, G. (2011). Die empirisch begründete Herleitung des Begriffs der „Interaktionalen Nische mathematischer Denkentwicklung“ (NMD). In B. Brandt, R. Vogel \& G. Krummheuer (Hrsg.), Die Projekte erStMaL und MaKreKi. Mathematikdidaktische Forschung am „Center for Individual Development and Adaptive Education" (S. 25-89). Münster: Waxmann.

Krummheuer, G. (2012). Die Interaktionsanalyse. In F. Heinzel (Hrsg.), Methoden der Kindheitsforschung: Ein Überblick über Forschungszugänge zur kindlichen Perspektive (S. 234-247). Weinheim, München: Beltz Juventa.

Krummheuer, G., \& Brandt, B. (2001). Paraphrase und Traduktion. Partizipationstheoretische Elemente einer Interaktionstheorie des Mathematiklernens in der Grundschule. Weinheim: Beltz.

Krummheuer, G., \& Naujok, N. (1999). Grundlagen und Beispiele Interpretativer Unterrichtsforschung. Opladen: Leske + Budrich. 
Miller, M. (1986). Kollektive Lernprozesse. Studien zur Grundlegung einer soziologischen Lerntheorie. Frankfurt am Main: Suhrkamp.

Newcombe, N. S., \& Huttenlocher, J. (2003). Making space. The development of spatial representation and reasoning. Cambridge: MIT Press.

Nührenbörger, M., \& Schwarzkopf, R. (2010). Die Entwicklung mathematischen Wissens in sozial-interaktiven Kontexten. In C. Böttinger, K. Bräuning, M. Nührenbörger, R. Schwarzkopf \& E. Söbbeke (Hrsg.), Mathematik im Denken der Kinder (S. 73-81). Seelze: Klett.

Oevermann, U., Allert, T., Konau, E., \& Krambeck, J. (1976). Die Methodologie einer „objektiven Hermeneutik" und ihre allgemeine forschungslogische Bedeutung in den Sozialwissenschaften. In H.G. Soeffner (Hrsg.), Interpretative Verfahren in den Sozial- und Textwissenschaften (S. 352-432). Stuttgart: Metzler.

Peter Koop, A., \& Grüßing, M. (2007). Bedeutung und Erwerb mathematischer Vorläuferfähigkeiten. In C. Brokmann-Nooren, I. Gereke, H. Kiper \& W. Renneberg (Hrsg.), Bildung und Lernen der Dreibis Achtjährigen (S. 153-166). Bad Heilbrunn: Klinkhardt.

Piaget, J. (1972). Das mathematische Denken. Stuttgart: Klett.

Rathgeb-Schnierer, E. (2015). Mathematische Bildung im Kindergarten. In B. Hauser, E. Rathgeb-Schnierer, R. Stebler \& F. Vogt (Hrsg.), Mehr ist mehr. Mathematische Frühförderung mit Regelspielen (S. 10-25). Seelze: Klett.

Reichertz, J. (2013). Die Abduktion in der qualitativen Sozialforschung. Wiesbaden: Springer VS.

Schneider, W., Küspert, P., \& Krajewski, K. (2016). Die Entwicklung mathematischer Kompetenzen. Paderborn: Ferdinand Schöningh.

Schütte, M. (2009). Sprache und Interaktion im Mathematikunterricht der Grundschule. Münster, New York, München, Berlin: Waxmann.

Schütte, M., \& Krummheuer, G. (2017). Mathematische Diskurse im Kindesalter - Der narratorische Diskurs. In Institut für Mathematik der Universität Potsdam (Hrsg.), Beiträge zum Mathematikunterricht (S. 877-880). Münster: WTM.

Schütte, M., Friesen, R.-A., \& Jung, J. (2019). Interactional analysis. A method for analysing mathematical learning processes in interactions. In G. Kaiser \& N. Presmeg (Hrsg.), Compendium for early career researchers in mathematics education. ICME-13 monograph (S. 101-129). Cham: Springer.

Schwarz, B. B., Neuman, Y., \& Biezuner, S. (2000). Two wrongs make a right? If they argue together! Cognition and Instruction, 18(4), 461-494.

Sfard, A. (2008). Thinking as communicating. Human development, the growth of discourses, and mathematizing. Cambridge: Cambridge University Press.

Steinbring, H. (2000). Mathematische Bedeutung als eine soziale Konstruktion - Grundzüge der epistemologisch orientierten mathematischen Interaktionsforschung. Journal für Mathematik-Didaktik, 21(1), $28-49$.

Strauss, A., \& Corbin, J. (1996). Grounded Theory. Grundlagen Qualitativer Sozialforschung. Weinheim: Beltz. Aus dem Amerikanischen von S. Niewiarra \& H. Legewie

Tiedemann, K. (2012). Mathematik in der Familie: Zur familialen Unterstützung früher mathematischer Lernprozesse in Vorlese- und Spielsituationen. Münster: Waxmann.

Vogel, R., \& Wippermann, S. (2005). Transferstrategien im Projekt VIB - Didaktische Design Pattern zur Dokumentation der Projektergebnisse. In C. Bescherer (Hrsg.), Einfluss der neuen Medien auf die Fachdidaktiken (S. 39-60). Baltmannsweiler: Schneider Verlag Hohengehren.

Vogler, A.M. (2020). Mathematiklernen im Kindergarten. Eine (mehrperspektivische) Untersuchung zu Chancen und Hürden beim frühen mathematischen Lernen in Erzieher*innen-Situationen. Münster: Waxmann.

Voigt, J. (1994). Entwicklung mathematischer Themen und Normen im Unterricht. In H. Maier \& J. Voigt (Hrsg.), Verstehen und Verständigung - Arbeiten zur interpretativen Unterrichtsforschung (S. 77-111). Köln: Aulis.

Voigt, J. (1995). Thematic patterns of interaction and sociomathematical norms. In P. Cobb \& H. Bauersfeld (Hrsg.), The emergence of mathematical meaning: interaction in classroom cultures (S. 163-201). Hillsdale: Lawrence Erlbaum.

Vygotsky, L. S. (1969). Denken und Sprechen. Frankfurt am Main: Fischer. 